\title{
Undrained expansion of a cylindrical cavity in clays with fabric anisotropy: theoretical solution
}

\author{
by \\ Nallathamby Sivasithamparam $\left({ }^{1}\right)$ and Jorge Castro $\left({ }^{2}\right)(*)$ \\ E-mail: $\left({ }^{1}\right)$ nallathamby.siva@ngi.no ; $\left(^{2}\right)$ castrogj@unican.es

\section{Affiliation:} \\ (1) Computational Geomechanics Division, Norwegian Geotechnical Institute, Oslo, \\ Norway \\ $\left({ }^{2}\right)$ Lecturer, Department of Ground Engineering and Materials Science, University of \\ Cantabria, Santander, Spain
}

$\left(^{*}\right)$ Corresponding author:

Group of Geotechnical Engineering

Department of Ground Engineering and Materials Science

University of Cantabria

Avda. de Los Castros, s/n

39005 Santander, Spain

Tel.: +34 942201813

Fax: +34 942201821

e-mail: castrogj@unican.es

Date: June 2017

Number of words: 6,100

Number of tables: 2

Number of figures: 15 


\begin{abstract}
This paper presents a novel, exact, semi-analytical solution for the quasi-static undrained expansion of a cylindrical cavity in soft soils with fabric anisotropy. This is the first theoretical solution of the undrained expansion of a cylindrical cavity under plane strain conditions for soft soils with anisotropic behaviour of plastic nature. The solution is rigorously developed in detail, introducing a new stress invariant to deal with the soil fabric. The semi-analytical solution requires numerical evaluation of a system of six first-order ordinary differential equations. The results agree with finite element analyses and show the influence of anisotropic plastic behaviour. The effective stresses at critical state are constant and they may be analytically related to the undrained shear strength. The initial vertical cross anisotropy caused by soil deposition changes towards a radial cross anisotropy after cavity expansion. The analysis of the stress paths shows that proper modelling of anisotropic plastic behaviour involves modelling not only the initial fabric anisotropy but also its evolution with plastic straining.
\end{abstract}

Keywords: Anisotropy, fabric of soils, clays, plasticity, stress path, theoretical analysis. 


\section{Introduction}

There is a wide variety of practical problems that may be modelled as the expansion (or contraction) of a spherical or cylindrical cavity in a solid mass. The mathematical solutions to those problems are usually categorized within the cavity expansion theory. The first solid mechanics applications of the cavity expansion theory were for metal indentation problems (e.g. [1, 12]). For geomaterials, the application came later [11] but has been widely investigated because of its utility in many practical situations. Some examples comprise the interpretation of in-situ tests like pressuremeter (e.g. [20,21]) or penetrometer tests (e.g. $[30,7])$ and the study of the installation disturbances caused by foundation elements like driven piles (e.g. [23]) or stone columns (e.g. [6]). It is also useful for wellbore instability and deep tunnels because, although in these cases the cavity is contracted instead of being expanded, the problem is similar mathematically (e.g. $[33,36,24])$. Here, the analysis limits to the quasi-static expansion of a cylindrical cavity in plane strain conditions because the solid mass is assumed as infinite. In clays, the cavity is usually expanded in a short period of time and then, no drainage is allowed (undrained conditions).

Cavity expansion solutions for soils under undrained conditions use mainly isotropic elastic-perfectly plastic models, using, for example, Tresca criterion (e.g. $[11,13,25])$, or isotropic hardening constitutive models, such as the Modified Cam Clay (MCC) model (e.g. $[4,8,9,26])$. There are some solutions for anisotropic materials within elasticity, e.g. cross-anisotropic material $[15,16]$, but, to the authors' knowledge, there were not any theoretical solutions for anisotropic behaviour of plastic nature. Only very recently, Li et al. [18] have published an analytical solution that accounts for an initial stress-induced anisotropy using a rotated yield surface. The rotated yield surface is fixed 
and, consequently, stresses near the cavity are not usually at a zero Lode's angle [22], which corresponds to plane strain conditions $\left(\sigma^{\prime}{ }_{2}=\left(\sigma^{\prime}{ }_{1}+\sigma^{\prime}{ }_{3}\right) / 2\right)$. This leads to unrealistic results in materials whose anisotropy evolves with plastic strains (e.g. clays).

This paper presents a novel, exact, semi-analytical solution of the undrained expansion of a cylindrical cavity in natural clays, which exhibit fabric anisotropy. The presented solution uses a constitutive model that considers anisotropy of plastic nature that evolves with plastic strains, both volumetric and deviatoric strains. The solution goes a step further than Li et al. [18] by introducing the evolution of anisotropy with plastic strains. The solution is semi-analytical as it requires the numerical evaluation of a system of six first-order ordinary differential equations. The results show the influence of the anisotropic plastic behaviour. Soil initial fabric is generated by soil deposition and consolidation due to the vertical compression caused by the gravity acceleration (vertical axis), whereas during cylindrical cavity expansion, the soil is compressed radially and its fabric anisotropy changes accordingly (radial axis).

The paper reviews existing analytical solutions of the cylindrical cavity expansion problem (Section 2) and details the assumptions and constitutive model used to develop the present solution (Section 3). Next, the full analytical development and its solution procedure are presented (Section 4). Boston blue clay, whose properties are depicted in Section 5, is the soft clay used for validation against finite element analyses, which are presented in Section 6. Finally, the results using the semi-analytical solution are discussed (Section 7) and some conclusions are derived.

\section{Undrained cavity expansion theory in soft soils}


By definition, the volumetric strain is null under undrained conditions. If additionally, the direction of the displacement vector is known at each point, only its value being unknown, then, the strain field is independent of the stresses and of the constitutive model and may be obtained using the boundary conditions. This happens, for example, for the present case of the expansion of a cylindrical cavity in plane strain conditions, in which the displacement vector at any point is horizontal and passes through the axis of symmetry. Initial stresses and material properties must also satisfy those symmetry conditions. So, the problem reduces to a one-dimensional boundary value problem. The strain field is first obtained from the incompressibility condition, and then the constitutive law is used to derive the effective stresses. Finally, equilibrium conditions may be imposed to get the internal pressure of the cavity.

A quite comprehensive review of solutions for different constitutive models may be found in $\mathrm{Yu}$ [35]. For critical state models, Collins \& Yu [9] developed a general approximate large-strain solution for both original and modified Cam Clay models. For the original Cam Clay model, they found a closed form solution, while for the modified Cam Clay model numerical integration is needed. As pointed out by Silvestri \& AbuSamra [26], Collins \& Yu [9] solution is approximate because it uses a simplified definition of the deviatoric stress, $q$, as will be explained in the next section. Chen $\&$ Abousleiman [8] were the first ones to obtain an exact analytical solution using the rigorous definition of the deviatoric stress, $q$, and a shear modulus, $G$, that varies with the mean pressure, $p^{\prime}$. Vrakas [31] developed a general exact solution for different Cam-clay models and presented a critical evaluation of the various simplifying expressions used for stress invariants. 


\section{Definition of the problem}

\subsection{Geometry and assumptions}

The quasi-static expansion of a cylindrical cavity of initial radius $a_{0}$ is studied. The axis of the cylindrical cavity is assumed as the vertical axis and the initial stress state is homogeneous and consists of a horizontal effective stress and a vertical effective stress $\left(\sigma^{\prime} H, \sigma^{\prime}\right)$. The initial stress state may be also formulated in terms of total stresses considering the initial pore water pressure $\left(u 0, \sigma_{H}, \sigma_{V}\right)$. The initial horizontal stress on the cavity is also $\sigma_{H}$ and it increases up to $\sigma_{a}$, upon expanding the cavity to a final radius $a$ (Figure 1).

The soil constitutive model will be detailed below but, despite being anisotropic, it will have initial cross-anisotropy (transversely isotropic material) with the main axis being the vertical one. So, the problem has axial symmetry and, due to the initial uniform stress state and the infinite extent of the soil and the cavity, plane strain conditions hold. In this way, the strain field is easily obtained as for isotropic incompressible materials.

Cylindrical coordinates $(r, \theta, z)$ are used throughout the paper because they are principal directions for this problem. The equilibrium equation in the radial direction for cylindrical coordinates that are principal directions may be written as

$\frac{\partial \sigma_{r}}{\partial r}+\frac{\sigma_{r}-\sigma_{\theta}}{r}=0$

or using effective stresses as

$$
\frac{\partial \sigma_{r}^{\prime}}{\partial r}+\frac{\partial u}{\partial r}+\frac{\sigma_{r}^{\prime}-\sigma_{\theta}^{\prime}}{r}=0
$$


Initially, the clay may be normally consolidated or overconsolidated, i.e. in a plastic state or inside the elastic region. For the latter case, the entire soil may remain elastic after expanding the cavity or there may be a plastic annulus around the cavity. Its external radius will be denoted as $r_{p}$ and defines the current elastic/plastic boundary (Figure 1). Obviously, if the cavity is further expanded, the value of $r_{p}$ gradually increases.

The cylindrical cavity expansion problem is an appropriate example of the importance of accounting for large displacements [32]. For large displacements, the internal cavity pressure approaches an asymptotic limit value, while for small displacements, the internal pressure continuously increases because it is considered to be applied on a cylinder with a smaller diameter $\left(a_{0}\right)$ than the real one $(a)$. In this paper, the material incompressibility makes it easier to account for large displacements. So, the current position of an arbitrary point, $r_{x}$, is directly related to the initial position of the point, $r_{x}$, and the initial and current radii of the cavity, $a_{0}$ and $a$, respectively

$r_{x}^{2}-a^{2}=r_{x 0}^{2}-a_{0}^{2}$

or, using dimensionless terms

$$
\left(\frac{r_{x 0}}{a}\right)^{2}=\left(\frac{r_{x}}{a}\right)^{2}+\left(\frac{a_{0}}{a}\right)^{2}-1
$$

Large-strain deformation is considered in the plastic region using natural (or logarithmic) strains, but small-strain deformation is used in the elastic region. This simplifying assumption does not affect the results because in the elastic region, the strains are much smaller than in the plastic annulus. The solution by Vrakas [31] 
considers large-strain formulation also in the elastic zone, but the differences are negligible.

\subsection{Constitutive model}

Natural soft clays exhibit a significant degree of anisotropy in their fabric, which initially is derived from the shape of the clay platelets, deposition process and onedimensional consolidation. Fabric anisotropy of natural clays is modified due to subsequent irrecoverable straining (e.g. [34]). Reorientation of particles and changes in particle contacts, i.e. changes in fabric anisotropy, cause changes in the mechanical response of the soil. Additionally, the more the subsequent loading path differs from the loading path that has created the current anisotropy, as happens for the cylindrical cavity expansion problem, the more anisotropy changes [14]. Therefore, it is important to use a constitutive model able to reproduce not only the initial anisotropic behaviour of the soft clay but also its evolution with plastic straining. In this study, the S-CLAY1 model [34] is used. S-CLAY1 is a Cam clay-type model with an inclined yield surface to model inherent anisotropy and a rotational component of hardening to model the development or erasure of fabric anisotropy during plastic straining. For the simplified stress space of triaxial compression $\left(\sigma_{2}=\sigma_{3}\right)$ and for an initial cross-anisotropy fabric with the main axis being the vertical one (e.g. a vertically cut sample), the yield curve is a sheared ellipse [10]

$f_{y}=\left(q-\alpha p^{\prime}\right)^{2}-\left(M^{2}-\alpha^{2}\right)\left(p^{\prime}{ }_{m}-p^{\prime}\right) p^{\prime}=0$

where $q$ is a deviatoric stress $\left(q=\sigma_{1}-\sigma_{3}\right), p$ ' is the mean effective stress, $M$ is the critical state value of the stress ratio (where $\eta=q / p^{\prime}$ ) and $p^{\prime}{ }_{m}$ and $\alpha$ define the size and inclination of the yield curve, respectively (Figure 2). 
S-CLAY1 incorporates two hardening laws. The first describes the change of size of the yield curve, which is assumed to be related solely to plastic volumetric strain (as in MCC)

$d p^{\prime}{ }_{m}=\frac{v p^{\prime}{ }_{m} d \varepsilon_{v}^{p}}{\lambda-\kappa}$

where $v$ is the specific volume, $\lambda$ is the slope of the post-yield compression curve in the $v$ - $\ln p$ ' plane for a constant $\eta$ stress path involving no change of anisotropy (e.g. isotropic loading of an isotropic sample) and $\kappa$ is the slope of the swelling line in the compression plane. The second hardening law (rotational hardening) describes the change of inclination of the yield curve produced by plastic straining, both volumetric and shear strains.

$\left.d \alpha=\omega\left[\left(\frac{3 \eta}{4}-\alpha\right) d \varepsilon_{v}^{p}\right\rangle+\omega_{d}\left(\frac{\eta}{3}-\alpha\right) d \varepsilon_{d}^{p} \mid\right]$

where $\omega$ is a material constant that controls the absolute effectiveness of plastic strains in rotating the yield surface towards the target value. Similarly, $\omega_{d}$ controls the relative effectiveness of the deviatoric plastic strain, $d \varepsilon_{d}^{p}$, and the volumetric plastic strain, $d \varepsilon_{v}^{p}$.

This constitutive model is rate-independent, does not consider interpaticle bonding and the elastic behaviour within the yield surface is isotropic.

\subsection{Definition of invariants}

Current cavity expansion solutions for Cam Clay models are formulated using $p$ ' and $q$ because they do not consider material anisotropy. Some researchers, such as Collins \& $\mathrm{Yu}$ [9] and Cao et al. [4], use simplified definitions of $p^{\prime}=\left(\sigma_{r}^{\prime}+\sigma_{\theta}^{\prime}\right) / 2$ and $q=\sigma_{r}^{\prime}-$ $\sigma_{\theta}^{\prime}\left(\right.$ or $\left.q=\sqrt{3} / 2\left(\sigma_{r}^{\prime}-\sigma_{\theta}^{\prime}\right)\right)$. For the cylindrical cavity expansion problem in a Cam Clay material, Chen \& Abousleiman [8] are apparently the first ones to use the full 3-D definition: 
$p^{\prime}=\frac{\left(\sigma_{r}^{\prime}+\sigma_{\theta}^{\prime}+\sigma_{z}^{\prime}\right)}{3}$

$q=\sqrt{\frac{1}{2}\left\lfloor\left(\sigma_{r}^{\prime}-\sigma_{\theta}^{\prime}\right)^{2}+\left(\sigma_{r}^{\prime}-\sigma_{z}^{\prime}\right)^{2}+\left(\sigma_{\theta}^{\prime}-\sigma_{z}^{\prime}\right)^{2}\right\rfloor}$

For the sake of simplicity, S-CLAY1 has been presented in the previous section using the simplified triaxial compression stress space and $p$ ' and $q$. However, the cavity expansion problem in plane strain conditions produces stress paths out of the triaxial compression stress space and causes the yield surface to rotate out of it and the use of $q$ is no longer valid (as will be explained below).

The yield surface of the model (Figure 2) can be expressed in generalized form as

$f_{y}=\frac{3}{2}\left(\boldsymbol{\sigma}_{\boldsymbol{d}}^{\prime}-\boldsymbol{\alpha}_{d} p^{\prime}\right)^{T}\left(\boldsymbol{\sigma}_{\boldsymbol{d}}^{\prime}-\boldsymbol{\alpha}_{d} p^{\prime}\right)-\left(M^{2}-\boldsymbol{\alpha}_{d}^{T} \boldsymbol{\alpha}_{d}\right)\left(p_{m}^{\prime}-p^{\prime}\right) p^{\prime}$

where

$\boldsymbol{\sigma}_{\boldsymbol{d}}^{\prime}=\left[\begin{array}{lll}\sigma_{r}^{\prime}-p^{\prime} & \sigma_{\theta}^{\prime}-p^{\prime} & \sigma_{z}^{\prime}-p^{\prime}\end{array}\right]^{T}$

and

$\boldsymbol{\alpha}_{\boldsymbol{d}}=\left[\begin{array}{lll}\alpha_{r}-1 & \alpha_{\theta}-1 & \alpha_{z}-1\end{array}\right]^{T}$

Although some Lode's angle dependency may be incorporated in the model, here a constant value of $M$ is considered, and consequently, the critical state surface in the stress space coincides with the Drucker-Prager yield criterion. The polar coordinates are principal directions for this problem, and consequently, the shear components of both stress and fabric tensors are not considered (Eqs. 11 and 12).

In this paper, a new invariant $\bar{q}$ is proposed instead of $q$ to derive a mathematical formulation for the cavity expansion problem in anisotropic plastic materials. This new 
invariant makes the derivation of the solution possible without modifying the constitutive model.

$\bar{q}=\sqrt{\frac{3}{2} Q}$

where

$Q=\left(\boldsymbol{\sigma}_{\boldsymbol{d}}^{\prime}-\boldsymbol{\alpha}_{d} p^{\prime}\right)^{T}\left(\boldsymbol{\sigma}_{\boldsymbol{d}}^{\prime}-\boldsymbol{\alpha}_{d} p^{\prime}\right)=s_{r}^{2}+s_{\theta}^{2}+s_{z}^{2}$

and $s_{i}$ are the following deviatoric stresses

$s_{i}=\sigma_{i}^{\prime d}-\alpha_{i}^{d} p^{\prime}=\sigma_{i}^{\prime}-\left(\alpha_{i}^{d}+1\right) p^{\prime} \quad$ for $i=r, \theta, z$

and $\alpha_{i}^{d}$ are deviatoric components of the fabric tensor

$\alpha_{i}^{d}=\alpha_{i}-1 \quad$ for $i=r, \theta, z$

Then, the yield surface may be expressed in a similar form as isotropic Cam-clay models

$f_{y}=\bar{q}^{2}-\left(M^{2}-\alpha^{2}\right)\left(p_{m}^{\prime}-p^{\prime}\right) p^{\prime}$

To understand the necessity of defining the new invariant $\bar{q}$, it is useful to study the cross section of the yield surface with the $\pi$-plane (hydrostatic or constant $p$ ' plane). For isotropic Cam-clay models, the yield curve in the $\pi$-plane is a circle centred in the origin ( $p^{\prime}$ axis) and $q$ is the radial distance to the origin, which coincides with the radius of the yield surface (Figure 3). However, for S-CLAY1 the yield surface is centred in the $\alpha$ axis and the radius of the yield surface is no longer $q$ (Eq. 9), but $\bar{q}$ (Eq. 13).

\section{Analytical solution}

\subsection{Elastoplastic stiffness matrix}


The usual decomposition of the strain increment vector $d \boldsymbol{\varepsilon}$ in an elastic, reversible contribution $d \boldsymbol{\varepsilon}^{e}$, and a plastic, irreversible contribution $d \boldsymbol{\varepsilon}^{p}$, is used.

$d \varepsilon=d \varepsilon^{e}+d \varepsilon^{p}$

The increment of elastic strain, $d \boldsymbol{\varepsilon}^{e}$, is expressed in terms of the effective stress using Hooke's elastic constitutive law

$\left\{\begin{array}{l}d \varepsilon_{r}^{e} \\ d \varepsilon_{\theta}^{e} \\ d \varepsilon_{z}^{e}\end{array}\right\}=\frac{1}{E}\left[\begin{array}{ccc}1 & -v & -v \\ -v & 1 & -v \\ -v & -v & 1\end{array}\right] \cdot\left\{\begin{array}{l}d \sigma_{r}^{\prime} \\ d \sigma_{\theta}^{\prime} \\ d \sigma_{z}^{\prime}\end{array}\right\}$

where Young's moduli $E$ is defined in terms of shear modulus $G$ and Poisson's ratio $v$

$E=2 G(1+v)$

In S-CLAY1 model, $G$ depends on the current stress state and is given by

$G=\frac{3(1-2 v) v}{2(1+v) \kappa} p^{\prime}$

As the model considers an associated flow rule, the three components of the plastic strain increment, $d \boldsymbol{\varepsilon}^{p}$, are

$\left\{\begin{array}{l}d \varepsilon_{r}^{p} \\ d \varepsilon_{\theta}^{p} \\ d \varepsilon_{z}^{p}\end{array}\right\}=\Lambda \cdot\left\{\begin{array}{l}\frac{\partial f_{y}}{\partial \sigma_{r}^{\prime}} \\ \frac{\partial f_{y}}{\partial \sigma_{\theta}^{\prime}} \\ \frac{\partial f_{y}}{\partial \sigma_{z}^{\prime}}\end{array}\right\}$

where $d \varepsilon_{r}^{p}, d \varepsilon_{\theta}^{p}$ and $d \varepsilon_{z}^{p}$ are plastic strain increments in $r, \theta$ and $z$ directions, respectively, and $\Lambda$ is the plastic multiplier. Derivatives of the yield function in terms of stresses $\left(\frac{\partial f_{y}}{\partial \sigma_{i}^{\prime}}\right)$ are given in the Appendix I.

To derive the plastic multiplier, the consistency condition $\left(\dot{f}_{y}=0\right)$ can be applied to the yield surface such that stresses cannot exist outside the yield surface 
$\dot{f}_{y}=\frac{\partial f_{y}}{\partial \sigma_{r}^{\prime}} d \sigma_{r}^{\prime}+\frac{\partial f_{y}}{\partial \sigma_{\theta}^{\prime}} d \sigma_{\theta}^{\prime}+\frac{\partial f_{y}}{\partial \sigma_{z}^{\prime}} d \sigma_{z}^{\prime}+\frac{\partial f_{y}}{\partial p_{m}^{\prime}} d p_{m}^{\prime}+\frac{\partial f_{y}}{\partial \alpha_{\boldsymbol{d}}} d \boldsymbol{\alpha}_{\boldsymbol{d}}=0$

or in terms of plastic strains

$\dot{f}_{y}=\frac{\partial f_{y}}{\partial \sigma_{r}^{\prime}} d \sigma_{r}^{\prime}+\frac{\partial f_{y}}{\partial \sigma_{\theta}^{\prime}} d \sigma_{\theta}^{\prime}+\frac{\partial f_{y}}{\partial \sigma_{z}^{\prime}} d \sigma_{z}^{\prime}+\frac{\partial f_{y}}{\partial p_{m}^{\prime}} \frac{\partial p_{m}^{\prime}}{\partial \varepsilon_{v}^{p}} d \varepsilon_{v}^{p}+\frac{\partial f_{y}}{\partial \boldsymbol{\alpha}_{d}} \frac{\partial \boldsymbol{\alpha}_{d}}{\partial \varepsilon_{v}^{p}}\left\langle d \varepsilon_{v}^{p}\right\rangle+\frac{\partial f_{y}}{\partial \boldsymbol{\alpha}_{\boldsymbol{d}}} \frac{\partial \boldsymbol{\alpha}_{d}}{\partial \varepsilon_{d}^{p}}\left|d \varepsilon_{d}^{p}\right|=0$

where $d \varepsilon_{v}^{p}$, and $d \varepsilon_{d}^{p}$, are obtained under the associated flow rule:

$d \varepsilon_{v}^{p}=\Lambda \frac{\partial f_{y}}{\partial p^{\prime}}$

$d \varepsilon_{d}^{p}=\Lambda \sqrt{\frac{2}{3}\left\{\frac{\partial f_{y}}{\partial \sigma^{\prime}}\right\}^{T} \cdot\left\{\frac{\partial f_{y}}{\partial \sigma^{\prime} d}\right\}}$

By substituting Eq. (25) and Eq. (26) into Eq. (24), the plastic multiplier $\Lambda$ can be derived as

$$
\Lambda=-\frac{\frac{\partial f_{y}}{\partial \sigma_{r}^{\prime}} d \sigma_{r}^{\prime}+\frac{\partial f_{y}}{\partial \sigma_{\theta}^{\prime}} d \sigma_{\theta}^{\prime}+\frac{\partial f_{y}}{\partial \sigma_{z}^{\prime}} d \sigma_{z}^{\prime}}{\frac{\partial f_{y} \partial p_{m}^{\prime} \partial f_{y}}{\partial p_{m}^{\prime} \partial \varepsilon_{v}^{p} \partial p^{\prime}}+\left\{\frac{\partial f_{y}}{\partial \alpha_{d}}\right\}^{T}\left[\left\{\frac{\partial \alpha_{d}}{\partial \varepsilon_{v}^{p}}\right\} \cdot \cdot \frac{\partial f_{y}}{\partial p^{\prime}}\right\rangle+\left\{\left\{\frac{\partial \alpha_{d}}{\partial \varepsilon_{d}^{p}}\right\} \cdot \sqrt{\frac{2}{3}\left\{\frac{\partial f_{y}}{\partial \sigma_{d}^{\prime}}\right\} \cdot\left\{\frac{\left.\partial f_{y}\right)}{\partial \sigma_{d}^{\prime}}\right\}}\right]}
$$

The plastic multiplier can be rewritten in a matrix form as

$$
\Lambda=\mathcal{H}\left[\begin{array}{lll}
\frac{\partial f_{y}}{\partial \sigma_{r}^{\prime}} & \frac{\partial f_{y}}{\partial \sigma_{\theta}^{\prime}} & \frac{\partial f_{y}}{\partial \sigma_{z}^{\prime}}
\end{array}\right]\left\{\begin{array}{l}
d \sigma_{r}^{\prime} \\
d \sigma_{\theta}^{\prime} \\
d \sigma_{z}^{\prime}
\end{array}\right\}
$$

where

$$
\mathcal{H}=-\frac{1}{\frac{\partial f_{y} \partial p_{m}^{\prime} \partial f_{y}}{\partial p_{m}^{\prime} \partial \varepsilon_{v}^{p} \partial p^{\prime}}+\left\{\frac{\partial f_{y}}{\partial \alpha^{d}}\right\}^{T}\left[\left\{\frac{\partial \alpha^{d}}{\partial \varepsilon_{v}^{p}}\right\} \cdot\left[\frac{\partial f_{y}}{\partial p^{\prime}}\right)+\left\{\frac{\partial \alpha^{d}}{\partial \varepsilon_{d}^{p}}\right\} \cdot \sqrt{\left.\frac{2\left(\frac{\partial f_{y}}{3}\right) \cdot\left(\frac{\partial f_{y}}{\partial \sigma^{\prime} d}\right)}{\partial \sigma^{\prime} d}\right\}}\right]}
$$

All required derivatives are given in the Appendix I. By substituting Eq. (28) into Eq. (22), the plastic strain increments can be obtained as

$$
\left\{\begin{array}{l}
d \varepsilon_{r}^{p} \\
d \varepsilon_{\theta}^{p} \\
d \varepsilon_{z}^{p}
\end{array}\right\}=\mathcal{H}\left[\begin{array}{ccc}
n_{r}^{2} & n_{r} n_{\theta} & n_{r} n_{z} \\
n_{\theta} n_{r} & n_{\theta}^{2} & n_{\theta} n_{z} \\
n_{z} n_{r} & n_{z} n_{\theta} & n_{z}^{2}
\end{array}\right] \cdot\left\{\begin{array}{l}
d \sigma_{r}^{\prime} \\
d \sigma_{\theta}^{\prime} \\
d \sigma_{z}^{\prime}
\end{array}\right\}
$$


where notations in Eq. (29) are defined as

$n_{i}=\frac{\partial f_{y}}{\partial \sigma_{i}^{\prime}}$ for $i=r, \theta, z$

Eq. (19) and Eq. (29) are combined in Eq. (18) to obtain the elasto-plastic constitutive equation

$\left\{\begin{array}{l}d \varepsilon_{r} \\ d \varepsilon_{\theta} \\ d \varepsilon_{z}\end{array}\right\}=\left[\begin{array}{ccc}\frac{1}{E}+\mathcal{H} n_{r}^{2} & -\frac{v}{E}+\mathcal{H} n_{r} n_{\theta} & -\frac{v}{E}+\mathcal{H} n_{r} n_{z} \\ -\frac{v}{E}+\mathcal{H} n_{\theta} n_{r} & \frac{1}{E}+\mathcal{H} n_{\theta}^{2} & -\frac{v}{E}+\mathcal{H} n_{\theta} n_{z} \\ -\frac{v}{E}+\mathcal{H} n_{z} n_{r} & -\frac{v}{E}+\mathcal{H} n_{z} n_{\theta} & \frac{1}{E}+\mathcal{H} n_{z}^{2}\end{array}\right] \cdot\left\{\begin{array}{l}d \sigma_{r}^{\prime} \\ d \sigma_{\theta}^{\prime} \\ d \sigma_{z}^{\prime}\end{array}\right\}$

Since the strains will be first obtained and then, the constitutive equation will be used to get the effective stresses, inversion of Eq. (31) is required

$\left\{\begin{array}{l}d \sigma_{r}^{\prime} \\ d \sigma_{\theta}^{\prime} \\ d \sigma_{z}^{\prime}\end{array}\right\}=\frac{1}{\Gamma}\left[\begin{array}{lll}c_{11} & c_{12} & c_{13} \\ c_{21} & c_{22} & c_{23} \\ c_{31} & c_{32} & c_{33}\end{array}\right] \cdot\left\{\begin{array}{l}d \varepsilon_{r} \\ d \varepsilon_{\theta} \\ d \varepsilon_{z}\end{array}\right\}$

where

$$
\begin{aligned}
& c_{11}=\frac{1}{E^{2}}\left(1-v^{2}+E \mathcal{H} n_{\theta}^{2}+2 E v \mathcal{H} n_{\theta} n_{z}+E \mathcal{H} n_{z}^{2}\right) \\
& c_{12}=c_{21}=\frac{1}{E^{2}}\left[-E \mathcal{H}\left(n_{\theta}+v n_{z}\right)+v\left(1+v-E \mathcal{H} n_{\theta} n_{z}+E \mathcal{H} n_{z}^{2}\right)\right] \\
& c_{13}=c_{31}=\frac{1}{E^{2}}\left[-E \mathcal{H} n_{r}\left(v n_{\theta}+n_{z}\right)+v\left(1+v-E \mathcal{H} n_{\theta}^{2}+E \mathcal{H} n_{\theta} n_{z}\right)\right] \\
& c_{22}=\frac{1}{E^{2}}\left(1-v^{2}+E \mathcal{H} n_{r}^{2}+2 E v \mathcal{H} n_{r} n_{z}+E \mathcal{H} n_{z}^{2}\right) \\
& c_{23}=c_{32}=\frac{1}{E^{2}}\left[v+v^{2}+E \mathcal{H} v n_{r}^{2}-E \mathcal{H} n_{\theta} n_{z}-E \mathcal{H} v n_{r}\left(n_{\theta}+n_{z}\right)\right] \\
& c_{33}=\frac{1}{E^{2}}\left(1-v^{2}+E \mathcal{H} n_{r}^{2}+2 E v \mathcal{H} n_{r} n_{\theta}+E \mathcal{H} n_{\theta}^{2}\right) \\
& \Gamma= \\
& -\frac{1+v}{E^{3}}\left[\begin{array}{c}
\left(-1+v+2 v^{2}\right)+E \mathcal{H}(-1+v) n_{r}^{2}+E \mathcal{H}(-1+v) n_{\theta}^{2}-2 E \mathcal{H} v n_{\theta} n_{z}-E \mathcal{H} n_{z}^{2} \\
+E \mathcal{H} v n_{z}^{2}-2 E \mathcal{H} v n_{r}\left(n_{\theta}+n_{z}\right)
\end{array}\right]
\end{aligned}
$$

\subsection{Rotational hardening rule}


The rotational hardening rule of S-CLAY1 [34] gives the change of the fabric components ( $d \alpha_{r}^{d}, d \alpha_{\theta}^{d}$ and $\left.d \alpha_{z}^{d}\right)$

$d \alpha_{i}^{d}=\omega\left(\left(\frac{3\left(\sigma_{i}^{\prime}-p^{\prime}\right)}{4 p^{\prime}}-\alpha_{i}^{d}\right)\left\langle d \varepsilon_{v}^{p}\right\rangle+\omega_{d}\left(\frac{\left(\sigma_{i}^{\prime}-p^{\prime}\right)}{3 p^{\prime}}-\alpha_{i}^{d}\right)\left|d \varepsilon_{d}^{p}\right|\right) \quad$ for $i=r, \theta, Z$

By substituting Eq. (25) and Eq. (26) into Eq. (33), the changes of the fabric components in terms of the plastic multiplier are obtained.

$d \alpha_{i}^{d}=\Phi_{i} \Lambda \quad$ for $i=r, \theta, z$

where

$\Phi_{i}=\omega\left(\left(\frac{3\left(\sigma_{i}^{\prime}-p^{\prime}\right)}{4 p^{\prime}}-\alpha_{i}^{d}\right)\left\langle\frac{\partial f_{y}}{\partial p^{\prime}}\right\rangle+\omega_{d}\left(\frac{\left(\sigma_{i}^{\prime}-p^{\prime}\right)}{3 p^{\prime}}-\alpha_{i}^{d}\right) \sqrt{\frac{2}{3}\left\{\frac{\partial f_{y}}{\partial \sigma^{\prime} d}\right\} \cdot\left\{\frac{\partial f_{y}}{\partial \sigma^{\prime} d}\right\}}\right)$ for $i=r, \theta, z$

The partial derivative of Eq. (34) with the radial direction provides the changes in the fabric components with the radial direction

$\frac{d \alpha_{i}^{d}}{d r}=\Phi_{i} \frac{\mathrm{d} \Lambda}{d r}$ for $i=r, \theta, z$

From Eq. (28), $\frac{\mathrm{d} \Lambda}{d r}$ can be obtained as

$\frac{\mathrm{d} \Lambda}{d r}=\mathcal{H}\left(n_{r} \frac{d \sigma_{r}^{\prime}}{d r}+n_{\theta} \frac{d \sigma_{\theta}^{\prime}}{d r}+n_{r} \frac{d \sigma_{z}^{\prime}}{d r}\right)$

By substituting Eq. (36) into Eq. (35), the changes in the fabric components with the radial direction are

$\frac{d \alpha_{i}^{d}}{d r}=\Phi_{i} \mathcal{H}\left(n_{r} \frac{d \sigma_{r}^{\prime}}{d r}+n_{\theta} \frac{d \sigma_{\theta}^{\prime}}{d r}+n_{r} \frac{d \sigma_{z}^{\prime}}{d r}\right) \quad$ for $i=r, \theta, z$

\subsection{Governing equations}

As the solution for the elastic zone is already known (e.g. [35]) and given in Appendix II, here, the governing equations are derived just for the plastic zone. The deformation 
in the plastic zone should be considered as a large strain problem (e.g. [9]); so, the radial and tangential strain increments can be defined in natural strain form as

$d \varepsilon_{r}=-\frac{\partial(d r)}{\partial r}$

$d \varepsilon_{\theta}=\frac{d r}{r}$

where $r$ and $d r$ are position of a material particle in the radial direction and change in the position of that particle, respectively.

Under undrained and plane strain conditions, the volumetric and vertical strains are zero, i.e. $d \varepsilon_{v}=d \varepsilon_{z}=0$.

$d \varepsilon_{r}=-d \varepsilon_{\theta}=\frac{d r}{r}$

By substituting Eq. (40) into Eq. (32) and applying plane strain conditions, i.e. $d \varepsilon_{z}=0$, the following differential equations are found.

$\frac{d \sigma_{r}^{\prime}}{d r}-\frac{c_{11}-c_{12}}{\Gamma} \frac{1}{r}=0$

$\frac{d \sigma_{\theta}^{\prime}}{d r}-\frac{c_{21}-c_{22}}{\Gamma} \frac{1}{r}=0$

$\frac{d \sigma_{z}^{\prime}}{d r}-\frac{c_{31}-c_{32}}{\Gamma} \frac{1}{r}=0$

$\frac{d \alpha_{z}^{d}}{d r}-\frac{\Phi_{z} \mathcal{H}}{\Gamma}\left[n_{r}\left(c_{11}-c_{12}\right)+n_{\theta}\left(c_{21}-c_{22}\right)+n_{r}\left(c_{31}-c_{32}\right)\right] \frac{1}{r}=0$ 
So, Eq. (41) and Eq. (42) provide the system of six first-order ordinary differential equations (ODE) that governs the problem in the plastic region. To solve the system, initial values (i.e. boundary conditions) and numerical integration are required. Initial values are those corresponding to the elastic/plastic boundary (as provided in the following section) and numerical integration is performed in the radial direction from $r_{x p}$ to $r_{x}$. Here, $r_{x}$ is the position of any particle located in the plastic zone and $r_{x p}$ is the position of that particle when it was just entering into the plastic state.

\subsection{Elastic/plastic boundary}

The initial values at the elastic/plastic boundary that have to be determined are the position of that boundary, $r_{x p}$, the corresponding stresses $\sigma_{r p}^{\prime}, \sigma_{\theta p}^{\prime}$, and $\sigma_{z p}^{\prime}$, and the corresponding fabric tensor, which is the initial one $\left(\alpha_{r 0}, \alpha_{\theta 0}, \alpha_{z 0}\right)$ as it does not change in the elastic zone. The elastic/plastic boundary (i.e. initial yield surface) can be defined using an isotropic overconsolidation ratio, $R$, in terms of mean effective stresses as

$R=\frac{p_{m}^{\prime}}{p_{i}^{\prime}}$

where $p_{m}^{\prime}$ is a preconsolidation mean stress (see Figure 2) and $p_{i}^{\prime}$ is an initial mean stress that may be obtained using the initial stress state $\left(p^{\prime}{ }_{0}\right.$ and $\left.q_{0}\right)$ and the yield surface (Eq. 5). Note that $R$ and the traditional overconsolidation ratio (OCR), which is expressed in terms of effective vertical stresses, are interrelated.

By using the coefficient of earth pressure at rest, $K_{0}$, the initial effective stresses can be written as 
$\sigma_{r 0}^{\prime}=\sigma_{\theta 0}^{\prime}=\frac{3 K_{0}}{1+2 K_{0}} p_{0}^{\prime}$ and $\sigma_{z 0}^{\prime}=\frac{3}{1+2 K_{0}} p_{0}^{\prime}$

Deviatoric stress at initial yielding, $q$ (Eq. 9), can be derived as shown in Figure 3 as

$q=\sqrt{q_{0}^{2}+\left(\bar{q}^{2}-\left(q_{0}-\alpha p_{0}^{\prime}\right)^{2}\right)}$

where

$q_{0}=\sqrt{\frac{3}{2}\left(\left(\sigma_{r 0}^{\prime}-p_{0}^{\prime}\right)^{2}+\left(\sigma_{\theta 0}^{\prime}-p_{0}^{\prime}\right)^{2}+\left(\sigma_{z 0}^{\prime}-p_{0}^{\prime}\right)^{2}\right)}$

and

$\bar{q}=\sqrt{\left(M^{2}-\alpha_{0}^{2}\right)\left(p_{m}^{\prime}-p_{0}^{\prime}\right) p^{\prime}}$

Using the initial stress state $\left(K_{0}\right.$ and $\left.p_{0}^{\prime}\right), R$ and $q$ (Eq. 45$)$, the stress state at the elastic/plastic boundary (i.e. initial yielding) can be derived as

$\sigma_{z p}^{\prime}=\frac{3}{1+2 K_{0}} p_{0}^{\prime}$

$\sigma_{r p}^{\prime}=\sigma_{r 0}^{\prime}+\sqrt{\frac{1}{3}\left(q^{2}-\left(\sigma_{z 0}^{\prime}-K_{0} \sigma_{z 0}^{\prime}\right)^{2}\right)}$

$\sigma_{\theta p}^{\prime}=\sigma_{\theta 0}^{\prime}-\sqrt{\frac{1}{3}\left(q^{2}-\left(\sigma_{z 0}^{\prime}-K_{0} \sigma_{z 0}^{\prime}\right)^{2}\right)}$

From the radial displacement given by Eq. (II.4) in Appendix II, the position of the material particle at the instant when the particle becomes plastic, $r_{x p}$, can be obtained as

$r_{x p}-r_{x 0}=\frac{\sigma_{r p}^{\prime}-\sigma_{r 0}^{\prime}}{2 G_{0}} r_{x p}$

where $r_{x 0}$ is the initial position of the particle and can be obtained using Eq. (4). So, $r_{x p}$ in terms of $r_{x}, a$ and $a_{0}$ can be determined substituting Eq. (4) into Eq. (49)

$\frac{r_{x p}}{a}=\frac{2 G_{0}}{2 G_{0}-\left(\sigma_{r p}^{\prime}-\sigma_{r 0}^{\prime}\right)} \sqrt{\left(\frac{r_{x}}{a}\right)^{2}+\left(\frac{a_{0}}{a}\right)^{2}-1}$ 
The location of the current elastic/plastic interface $r_{p}$ can be obtained by equating both $r_{x p}$ and $r_{x}$ to $r_{p}$ in Eq. (50) because it corresponds to the particle that is just entering the plastic zone right now.

$\frac{r_{p}}{a}=\sqrt{\left(\frac{a_{0}}{a}\right)^{2}-1} / \sqrt{\left(\frac{\sigma_{r p}^{\prime}-\sigma_{r 0}^{\prime}}{2 G_{0}}\right)^{2}-\frac{\sigma_{r p}^{\prime}-\sigma_{r 0}^{\prime}}{G_{0}}}$

\subsection{Excess pore pressures}

The excess pore pressure, $\Delta u$, at point $r_{x}$ can be calculated applying equilibrium of radial stresses, i.e. by integrating Eq. (2) from the elastic/plastic interface up to the point $r_{x}$ as

$\Delta u=u\left(r_{x}\right)-u_{0}=\sigma_{r p}^{\prime}-\sigma_{r}^{\prime}\left(r_{x}\right)-\int_{r_{p}}^{r_{x}} \frac{\sigma_{r}^{\prime}-\sigma_{\theta}^{\prime}}{r} d r$

\subsection{Solution procedure}

Stresses, changes in fabric anisotropy, excess pore pressures around the cavity and other results presented below were obtained using a standard differential solver available in GNU Octave v4.0. An existing solver 'lsode' was utilized to solve the system of six first-order ordinary differential equations derived in Eqs. (41) and (42). Figure 4 summarizes the solution procedure used here to solve the cavity expansion problem.

\section{Boston Blue clay}

To validate the analytical solution and to illustrate its application, Boston Blue clay was chosen since it is a well-documented clay that has already been used in previous cylindrical cavity expansion studies (e.g. $[8,23])$. The soil parameters are detailed in Table 1. For the sake of comparison, basic Cam clay parameters are taken from Chen \& 
Abousleiman [8]. Additional anisotropy parameters are deduced following Wheeler et al. [34]; for example, the initial inclination of the yield surface, $\alpha_{0}$, is deduced from the stress history of the soil (one dimensional compression) to fit the coefficient of lateral earth pressure at rest for normally consolidated conditions, $K_{0 \mathrm{NC}}$. OCR of Boston Blue clay varies with depth; so, the corresponding state parameters are shown in Table 2. $K_{0 \mathrm{NC}}$ is estimated using Jaky's expression:

$K_{0 N C}=1-\sin \phi=1-\frac{3 M}{6+M}$

where $\phi$ is the friction angle of the soil in triaxial compression.

For higher OCR values, $K_{0}$ is estimated by numerical simulation of the corresponding loading and unloading process starting from the normally consolidated case. $G_{0}$ is calculated using Eq. (21) and the undrained shear strength for triaxial compression, $c_{u, T X}$, is also analytically obtained from the previous values using the following expression, which was derived using a similar procedure as that used by Potts \& Zdravkovic [22] for MCC

$$
\frac{c_{u, T X}}{{\sigma_{z 0}^{\prime}}_{z 0}}=O C R \cdot M \frac{1+2 K_{0 N C}}{9}\left(1+B^{2}\right)\left[\frac{3\left(1+2 K_{0}\right)}{2\left(1+2 K_{0 N C}\right) O C R\left(1+B^{2}\right)}\right]^{\frac{\kappa}{\lambda}}
$$

where

$$
B=\frac{3\left(1-K_{0 N C}\right) /\left(1+2 K_{0 N C}\right)-\alpha_{0}}{\sqrt{M^{2}-\alpha_{0}^{2}}}
$$

In this case, failure is reached for plane strain conditions. and, consequently, the undrained shear strength is defined, according to Tresca failure criterion, for a zero Lode's angle, i.e. plane strain conditions (e.g. [5]):

$c_{u, P S}=2 / \sqrt{ } 3 c_{u, T X}$ 
However, for this anisotropic model, Eq. (55) only holds for normally consolidated conditions. When the soil is overconsolidated (OCR $>1$ ), the mean effective stress at critical state is different in triaxial compression $\left(\theta=-30^{\circ}\right)$ and in plane strain conditions because the $q$ values are different at yielding (horizontal path from the initial point) (see Figure 3). Consequently, Eq. (55) is no longer valid and $c_{u, P S}$ values had to be obtained numerically (Table 2).

For normally consolidated conditions $(\mathrm{OCR}=1)$, Eqs. (54) and (55) give values of $c_{u} / \sigma_{z 0}^{\prime}=0.31$ and 0.35 (Table 2), respectively, similar to those measured in the laboratory (0.33) for triaxial compression and plane strain compression [17].

\section{Validation}

To validate the semi-analytical solution, finite element simulations have been performed using the commercial code PLAXIS 2D 2015 [2]. The S-CLAY1 model has been implemented as User-defined soil model in PLAXIS, using an automatic substepping in combination with a modified Newton-Raphson integration scheme [27, 28].

The geometrical modelling of the cylindrical cavity expansion problem (Figure 5) is based on that proposed by Burd \& Houlsby [3] using a correcting layer. That is necessary because the semi-analytical solution assumes a material of infinite extent. In order to model that using a mesh of finite dimensions, a correcting layer is added to the perimeter of the mesh. Burd \& Houlsby [3] show that the properties of the correcting layer should satisfy the following relationship

$\frac{G_{c}}{G}=\frac{\left[\left(r_{c} / r_{e}\right)^{2}-1\right]\left(1-2 v_{c}\right)}{1+\left(r_{c} / r_{e}\right)^{2}\left(1-2 v_{c}\right)}$ 
where $G$ is the shear modulus of the material, $G_{c}$ and $v_{c}$ are the shear modulus and the Poisson's ratio of the correcting layer and $r_{c}$ and $r_{e}$ are the outer and inner radius of the correcting layer, respectively.

Here, common values of $v_{c}=0.3, r_{e}=65 a_{0}$ and $r_{c}=2 r_{e}$ were assumed and $G_{c}$ was calibrated using Eq. (56) and Eq. (21). Besides, $G_{c}$ was varied within a limited range to confirm that the results were not affected.

In the analytical solution, the clay is perfectly incompressible, while in the numerical simulations a high but finite value of the bulk modulus is considered. Parametric analyses were performed to confirm the negligible influence. Only, when very high values of the bulk modulus are used, the quality of the stresses is poor (they oscillate).

A prescribed displacement $\left(a-a_{0}\right)$ was imposed at the cavity and initial homogeneous stresses and clay properties were input. To account for large displacements, the numerical code uses an updated Lagrangian formulation [19] and adopts the corotational rate of Kirchhoff stress (also known as Hill stress rate). The details of the implementation can be found in Van Langen [29].

Comparison between finite element simulations and the semi-analytical solution gives negligible differences. For illustrative purposes, finite element results are shown in Figure 6a. To avoid duplication, finite element results have not been included in other figures. 


\section{Results and discussion}

\subsection{Internal cavity pressure}

The internal pressure (radial stress) necessary to expand the cavity, $\sigma_{a}$, is one of the important variables of the problem. Its value increases as the cavity is expanded. When the cavity has been notably expanded $\left(a_{/} a_{0}>2\right), \sigma_{a}$ approaches an asymptotic limit value, sometimes called pressuremeter limit pressure. Figure 7 shows its variation with the normalized cavity radius for different OCR values. For the sake of comparison, ambient pore pressures are not included in $\sigma_{a}$. Excess pore pressures at the cavity wall are also depicted in Figure 7. For high OCR values, slight negative excess pore pressures could be generated at the beginning of the cavity expansion (small $a / a_{0}$ values). The final limit values of $\sigma_{a}$ and $\Delta u(a)$ decrease with the OCR in a roughly logarithmic way as proposed by Randolph et al. [23].

The difference between $\sigma_{a}$ and $\Delta u(a)$, i.e. the radial effective stress $\sigma^{\prime}{ }_{r}$, quickly reaches a constant value that is independent of the OCR $\left(\sigma_{r}^{\prime}=(1+\sqrt{3} / M) c_{u}\right.$ for $\left.a / a_{0}>1.3\right)$ as explained in the next section.

\subsection{Stresses around the cavity}

Figure 6 shows the stresses around the cavity for different OCR values, namely 1, 1.5 and 5 , and when the cavity radius is twice the initial one $\left(a / a_{0}=2\right)$. The stresses are normalized by the undrained shear strength of each case for plane strain conditions (Table 2). Near the cavity, the stresses are constant with the radius because they are at critical state (CS). The extension of the CS region is smaller in this anisotropic solution $(r / a<2)$, than in previous isotropic solutions $(r / a>2)$, because important plastic strains are necessary to rotate the yield surface until its CS position. The CS region has been 
determined assuming a tolerance of $0.1 \%$ in any stress change and its extension is around $r / a=1.6$ for any OCR. For OCR=1, all the material points yield just when the cavity expansion starts, so there is no elastic region. The size of the elastic region increases with the OCR value. For high OCR values (for example, OCR=5), there are negative values not only of the excess pore pressure but also of the hoop effective stresses. Attention should be paid to this negative effective stresses because they may be not realistic depending on the allowed tensile stress in the soil.

The effective stresses at CS may be analytically obtained as a function of the undrained shear strength for plane strain conditions. The vertical stress is the mean value of the radial and hoop stresses because plane strain conditions hold and given that $q_{\digamma} \sqrt{ } 3 c_{u}=M \cdot p^{\prime} f$, the following values are obtained at CS:

$\sigma_{r}^{\prime}=(\sqrt{ } 3 / M+1) c_{u} \quad ; \quad \sigma^{\prime}=(\sqrt{ } 3 / M) c_{u} \quad ; \quad \sigma^{\prime}{ }_{\theta}=(\sqrt{ } 3 / M-1) c_{u}$

\subsection{Stress paths}

To provide an understanding of the cavity expansion problem and the role of the fabric anisotropy of the clay is important to analyze the stress paths followed by a point at the cavity wall. Points at further distances follow the same path but if they are outside the CS region, they stop earlier. Figure 8 shows the effective stress paths (ESP) in the $p^{\prime}-q$ diagram for $\mathrm{OCR}=1,1.5$ and 5 . As is common practice, the intersection of the yield surface (YS) with the triaxial plane is also depicted in Figure 8. It is worth noting that for isotropic yield surfaces, there is a unique representation in the $p^{\prime}-q$ diagram, but for anisotropic yield surfaces, it depends on the intersection plane. As the stress paths go outside the triaxial plane, the intersection of the yield surface with the triaxial plane, as plotted in Figure 8, is meaningless. For example, the stress paths in Figure 8b and 
Figure 8c go vertically upwards until they reach the yield surface and they bend when the yield surface is reached. As the yield surface is reached outside the triaxial plane, the $q$ value is lower than expected from the intersection of the yield surface with the triaxial plane plotted in Figure 8. Rotation of the yield surface also plays an important role. To perceive that, another type of stress space should be used, e.g. the $\pi$-plane (Figure 9).

The cross section of the yield surface with the $\pi$-plane is a circle, whose centre is in the $\alpha$-axis $\left(\alpha p^{\prime}\right)$ and its radius is $\bar{q}$. Within the elastic region, the ESP goes straight to the right until it reaches the yield surface (Figure $9 \mathrm{~b}$ and Figure $9 \mathrm{c}$ ) because $\sigma_{z}^{\prime}$ does not change and the amount that $\sigma_{r}{ }_{r}$ increases is the same as $\sigma^{\prime}{ }_{\theta}$ decreases. Once, the ESP touches the yield surface, it bends towards plane strain conditions $\left(\theta=0^{\circ}\right)$ $\left(\sigma^{\prime}{ }_{z}=\left(\sigma^{\prime}{ }_{r}+\sigma^{\prime} \theta\right) / 2\right)$ and the yield surface also rotates. The final point, i.e. at critical state, corresponds to $q_{f}=\sqrt{3} c_{u}$, is in plane strain $\left(\theta=0^{\circ}\right)$ and at the rightmost point of the yield surface in the $\pi$-plane. The center of the yield surface, i.e. the $\alpha$-axis, is also in the plane strain plane $\left(\theta=0^{\circ}\right)$ and the model (S-CLAY1) predicts a unique inclination of the yield surface at critical state, namely $\alpha=M / 3$. In Figure 9 a, the path followed by the $\alpha \cdot p$, vector is also plotted to highlight how the yield surface rotates towards a zero Lode's angle $\left(\theta=0^{\circ}\right)$.

When the $K_{0}$ line is below the $\alpha$-axis, i.e. high OCR values (e.g. Figure 9c), the ESP goes upward a bit after reaching the yield surface because it goes towards the rightmost point of the yield surface and the rotation of the yield surface is not instantaneous and follows with some delay. 


\subsection{Evolution of fabric anisotropy}

One of the main capabilities of the used constitutive model (S-CLAY1) is the possibility to reproduce the evolution of fabric anisotropy with plastic straining. So, fabric anisotropy changes during the cavity expansion process as already shown in Figure 9. If just the inclination of the yield surface changes, the analysis may be reduced to the scalar value $\alpha$, but if the yield surface also rotates as in this case, it is necessary to analyze the fabric tensor $\alpha$. Figure 10 shows the variation of the scalar value $\alpha$ (inclination of the $\alpha$-axis in a $p^{\prime}-q$ diagram) and the different components of the fabric tensor, $\alpha_{i}$. The inclination of the yield surface at critical state is $\alpha=M / 3$ and, since this inclination corresponds to plane strain conditions $\left(\alpha_{z}=\left(\alpha_{r}+\alpha_{\theta}\right) / 2\right)$, the fabric tensor at CS, i.e. near the cavity, is $\left[\begin{array}{lll}\alpha_{r} & \alpha_{\theta} & \alpha_{z}\end{array}\right]=\left[\begin{array}{lll}1+\sqrt{3} M / 9 & 1-\sqrt{3} M / 9 & 1\end{array}\right]$. The results for different OCR only vary in the plastic region, depending on its extent.

To help to visualize the changes in the soil fabric, Figure 11 shows the $\left(\alpha_{z}-\alpha \theta, \alpha_{r}-\alpha_{\theta}\right)$ vector in arbitrary points. This vector tries to resemble the orientation of the clay palettes and in this problem, it changes from horizontal direction for an initial vertical cross anisotropy towards a nearly vertical one for radial cross anisotropy after cavity expansion.

\subsection{Influence of anisotropy}

The initial inclination of the yield surface $\left(\alpha_{0}\right)$ has been varied to analyze its influence. Between the limit cases of $\alpha_{0}=0$ and $\alpha_{0}=0.46$, several values have been checked but the results are just intermediate cases and therefore, only the limit cases $\left(\alpha_{0}=0\right.$ and $\left.\alpha_{0}=0.46\right)$ are plotted in Figure 12. When normalized by $c_{u}$, the results at CS are the same as already demonstrated. So, the main differences are in the value of $c_{u}$, the excess pore 
pressures and their radial extent. For an initially inclined yield surface $\left(\alpha_{0}=0.46\right)$, the radial extent of the excess pore pressures is larger as the yield surface quickly starts to rotate and at the cavity boundary its normalized value is consequently higher. On the other hand, the rotational hardening rule causes that in both cases the final inclination of the yield surface is the same.

To really evaluate the influence of considering plastic anisotropy, the rotational hardening rule must be also deactivated $(\omega=0)$, yielding into the MCC model. For the MCC, the present solution exactly coincides with the solution by Chen \& Abousleiman [8]. Comparison between MCC and S-CLAY1 is depicted in Figure 13. When considering plastic anisotropy, the extend of the excess pore pressures is larger as the yield surface quickly starts to rotate but, on the other hand, this rotation reduces pore pressure generation and the maximum excess pore pressure at the cavity wall is lower. Effective stresses at CS are exactly the same as already justified. In the plastic zone, there are small differences, for example, the hoop stress monotonically decreases for MCC, while for S-CLAY1 it decreases and slightly increases near the CS zone due to rotation of the yield surface and the shape of the rotational hardening law (Eq. 7) [34].

\subsection{Case without rotational hardening}

The constitutive model used to develop the analytical solution (S-CLAY1) is able to reproduce not only the initial anisotropic behaviour of the clay but also its evolution with plastic straining. The present problem (cavity expansion) is a very good example of the importance of considering the changes in fabric anisotropy when the loading path notably differs from the loading path that created the initial anisotropy (one-dimensional compression). To highlight the differences, the rotational hardening law is deactivated 
but the soil is initially anisotropic $(\omega=0)$ ('no RH') and the results compared with the reference case where rotational hardening was activated ('with $\mathrm{RH}$ ') in the anisotropic soil.

Figure 14 shows the stress path at the cavity wall when rotational hardening is deactivated $\left(\mathrm{OCR}=1, \alpha_{0}=0.46\right)$. As for the case with $\mathrm{RH}$, the stress path goes towards a zero Lode's angle $\left(\theta=0^{\circ}\right)$ (Figure 14a). However, the stress path does not reach $\theta=0^{\circ}$ because the yield surface is inclined in the vertical axis and it cannot rotate towards $\theta=0^{\circ}$. So, the stress state must remain within the vertically inclined yield surface, which is not realistic. The final point of the stress path is at the rightmost point of the yield surface in the $\pi$-plane, which has the shape of a circle. In this case, the effective mean stress also decreases (Figure 14b) to allow the final point to be closer to $\theta=0^{\circ}$.

Neglecting fabric evolution with plastic strains in clays leads to unrealistic results (Figure 15), particularly in the most important one, the radial stress, which is notably underpredicted if rotational hardening is not allowed. So, cavity expansion problems in natural clays should not be analyzed with a plastic anisotropic model that does not consider rotational hardening, as done in $\mathrm{Li}$ et al. [18]. Since the formulation of the yield surface in Li et al. [18] is different from the S-CLAY1 model, the predictions are expected to be different from the present ones.

\section{Conclusions}

A semi-analytical exact solution for the undrained expansion of a cylindrical cavity in clays with fabric anisotropy is rigorously developed. The adopted constitutive model, 
namely S-CLAY1, is a Cam clay model that reproduces plastic anisotropy, both initial fabric and its evolution with plastic straining. To develop the solution, a new stress invariant $\bar{q}$, which represents the radius of the yield surface in the $\pi$-plane is introduced. The solution involves the numerical integration of a system of six first-order ordinary differential equations, three of them corresponding to the effective stresses in cylindrical coordinates and the other three to the components of the fabric tensor.

The semi-analytical solution is validated against finite element analyses, using Boston blue clay as the reference clay. After cavity expansion, three zones may generally be distinguished: the external elastic zone, the intermediate plastic zone and the internal critical state zone. Considering soil plastic anisotropy leads to a larger extension of the intermediate plastic zone as the yield surface is reached earlier. When the stress path reaches the yield surface, excess pore pressures start to develop and the yield surface rotates towards a zero Lode's angle. For high OCR, slight negative pore pressures may develop. At CS, the effective stresses are constant and their values may be analytically obtained as a function of $c_{u}$. S-CLAY1 predicts a unique fabric tensor at CS for plane strain conditions, which may be analytically obtained. The initial vertical cross anisotropy caused by the soil deposition and consolidation changes towards a radial cross anisotropy after cavity expansion. Normalised maximum excess pore pressures at cavity wall are slightly lower for S-CLAY1 than for MCC.

Analytical solutions for soils with fabric anisotropy must consider fabric evolution with plastic straining because otherwise stresses near the cavity are not usually at a zero Lode's angle, which leads to unrealistic results. 


\section{Acknowledgements}

The research was initiated as part of GEO-INSTALL (Modelling Installation Effects in Geotechnical Engineering, PIAP-GA-2009-230638) and CREEP (Creep of Geomaterials, PIAP-GA-2011-286397) projects supported by the European Community through the programme Marie Curie Industry-Academia Partnerships and Pathways (IAPP) under the $7^{\text {th }}$ Framework Programme. 


\section{References}

1. Bishop RF, Hill R, Mott NF (1945) The theory of indentation hardness tests. Proc Phys Soc 57:147-159

2. Brinkgreve RBJ, Kumarswamy S, Swolfs WM (2015) Plaxis 2D 2015 Manual. Plaxis bv, the Netherlands

3. Burd HJ, Houlsby GT (1990) Finite element analysis of two cylindrical expansion problems involving nearly incompressible material behavior. Int $\mathrm{J}$ Num Anal Methods Geomech 14:351-366

4. Cao LF, The CI, Chang MF (2001) Undrained cavity expansion in modified Cam clay I: theoretical analysis. Géotechnique 51:323-334

5. Carter JP, Booker JR, Yeung SK (1986) Cavity expansion in cohesive frictional soils. Géotechnique 36:345-358

6. Castro J, Karstunen M (2010) Numerical simulations of stone column installation. Can Geotech J 47:1127-1138

7. Ceccato F, Simonini P (2017) Numerical study of partially drained penetration and pore pressure dissipation in piezocone test. Acta Geotechnica 12:195-209

8. Chen SL, Abousleiman YN (2012) Exact undrained elasto-plastic solution for cylindrical cavity expansion in modified Cam Clay soil. Géotechnique 62:447-456

9. Collins IF, Yu HS (1996) Undrained cavity exapnsions in critical state soils. Int J Num Anal Methods Geomech 20(7):489-516

10. Dafalias YF (1986) An anisotropic critical state soil plasticity model. Mech Res Commun 13:341-347

11. Gibson RE, Anderson WF (1961) In situ measurement of soil properties with the pressuremeter. Civ Eng Publ Works Rev 56:615-618 
12. Hill R, Mott NF, Pack DC (1944) Penetration of Munroe Jets. Armament Res Dept, Report 2/44, UK

13. Hill R (1950) The mathematical theory of plasticity. Oxford University Press

14. Karstunen M, Koskinen M (2008) Plastic anisotropy of soft reconstituted clays. Can Geotech J 45(3):314-328

15. Kolymbas D, Wagner P, Blioumi A (2012) Cavity expansion in cross-anisotropic rock. Int J Num Anal Methods Geomech 36:128-139

16. Lekhnitskii SG (1963) Theory of elasticity of an anisotropic elastic body. HoldenDay, Inc

17. Levadoux J-N (1980) Pore pressures in clays due to cone penetration. PhD Thesis, Dept Civil Eng, Mass Inst Tech

18. Li L, Li J, Sun D (2016) Anisotropically elasto-plastic solution to undrained cylindrical cavity expansion in $K_{0}$-consolidated clay. Comput Geotech 73:83-90

19. McMeeking RM, Rice JR (1975) Finite-element formulation for problems of large elastic-plastic deformation. Int J Solids Struct 11:601-616

20. Palmer AC (1972) Undrained plane-strain expansion of a cylindrical cavity in clay: A simple interpretation of the pressuremeter test. Géotechnique 22:451-457

21. Prevost JH, Hoeg K (1975) Analysis of pressuremeter in strain softening soil. J Geotech Eng Div, ASCE 101:717-732

22. Potts DM, Zdravkovic L (1999) Finite element analysis in geotechnical engineering: theory. Thomas Telford, London

23. Randolph MF, Carter JP, Wroth CP (1979) Driven piles in clay-the effects of installation and subsequent consolidation. Géotechnique 29:361-393 
24. Rott J, Mašín D, Boháč J, Krupička M, Mohyla T (2015) Evaluation of K0 in stiff clay by back-analysis of convergence measurements from unsupported cylindrical cavity, Acta Geotechnica 10 719-733

25. Shuttle D (2007) Cylindrical cavity expansion and contraction in Tresca soil. Géotechnique 57(3):305-308

26. Silvestri V, Abou-Samra G (2011) Application of the exact constitutive relationship of modified Cam clay to the undrained expansion of a spherical cavity. Int J Num Anal Meth Geomech 35:53-66

27. Sivasithamparam N (2012) Development and implementation of advanced soft soil models in finite elements. $\mathrm{PhD}$ thesis, University of Strathlcyde, Glasgow

28. Sivasithamparam N, Castro J (2016) An anisotropic elastoplastic model for soft clays based on logarithmic contractancy. Int J Num Anal Methods Geomech 40:596-621

29. Van Langen H (1991) Numerical analysis of soil-structure interaction. Ph.D. thesis, Delft University of Technology, Delft, the Netherlands

30. Vesic AS (1972) Expansion of cavities in infinite soil mass. J Soil Mech Found Div, ASCE 98:265-290

31. Vrakas A (2016a) A rigorous semi-analytical solution for undrained cylindrical cavity expansion in critical state soils. Int $\mathrm{J}$ Num Anal Methods Geomech 40(15):2137-2160

32. Vrakas A (2016b) Relationship between small and large strain solutions for general cavity expansion problems in elasto-plastic soils. Comput Geotech 76:147-153

33. Vrakas A, Anagnostou G (2015). Finite strain elastoplastic solutions for the undrained ground response curve in tunnelling. Int J Numer Anal Meth Geomech 39: $738-761$ 
34. Wheeler SJ, Naatanen A, Karstunen M, Lojander M (2003) An anisotropic elastoplastic model for soft clays. Can Geotech J 40(2):403-418

35. Yu HS (2000) Cavity expansion methods in geomechanics. Kluwer Academic, Dordrecht

36. Yu HS, Rowe RK (1999) Plasticity solutions for soil behaviour around contracting cavities and tunnels. Int J Num Anal Methods Geomech 23:1245-1279 


\section{APPENDIX I: Derivatives}

The partial derivatives used in the analytical solution are

$\frac{\partial f_{y}}{\partial \sigma_{i}^{\prime}}=\frac{p^{\prime}\left(M^{2}-\alpha^{2}-\bar{\eta}^{2}\right)}{3}+\left(3 s_{i}-s_{r} \alpha_{r}^{d}-s_{\theta} \alpha_{\theta}^{d}-s_{z} \alpha_{z}^{d}\right)$ for $i=r, \theta, z$

where

$\bar{\eta}=\frac{\bar{q}}{p \prime}$

$\bar{q}=\sqrt{\frac{3}{2} Q}$

and

$\frac{\partial f_{y}}{\partial p_{m}^{\prime}}=-p^{\prime}\left(M^{2}-\alpha^{2}\right)$

$\frac{\partial p_{m}^{\prime}}{\partial \varepsilon_{v}^{p}}=\frac{v p^{\prime}}{(\lambda-\kappa)\left(M^{2}-\alpha^{2}\right)}\left(M^{2}-\alpha^{2}+\bar{\eta}^{2}\right)$

$\frac{\partial f_{y}}{\partial p^{\prime}}=p^{\prime}\left(M^{2}-\alpha^{2}-\bar{\eta}^{2}\right)-3\left(s_{r} \alpha_{r}^{d}+s_{\theta} \alpha_{\theta}^{d}+s_{z} \alpha_{z}^{d}\right)$

$\frac{\partial f_{y}}{\partial \alpha_{i}^{d}}=-3 s_{i} p^{\prime}+3 \alpha_{i}^{d} \frac{\bar{q}^{2}}{M^{2}-\alpha^{2}} \quad$ for $i=r, \theta, z$

$\frac{\partial f_{y}}{\partial \sigma_{i}^{\prime d}}=3 s_{i} \quad$ for $i=r, \theta, z$

$\frac{\partial \alpha_{i}^{d}}{\partial \varepsilon_{v}^{p}}=\omega\left(\frac{3\left(\sigma_{i}^{\prime}-p^{\prime}\right)}{4 p^{\prime}}-\alpha_{i}^{d}\right) \quad$ for $i=r, \theta, z$

$\frac{\partial \alpha_{i}^{d}}{\partial \varepsilon_{d}^{p}}=\omega \omega_{d}\left(\frac{\left(\sigma_{i}^{\prime}-p^{\prime}\right)}{3 p^{\prime}}-\alpha_{i}^{d}\right)$ for $i=r, \theta, z$

$\sqrt{\frac{2}{3}\left\{\frac{\partial f_{y}}{\partial \sigma^{\prime}}\right\} \cdot\left\{\frac{\partial f_{y}}{\partial \sigma^{\prime}}\right\}}=2 \bar{q}$ 


\section{APPENDIX II: Elastic solution}

The solution for the elastic total stresses $\left(\sigma_{r}, \sigma_{\theta}, \sigma_{z}\right)$ and the radial displacement $\left(u_{r}\right)$ can be obtained imposing the assumption of total volumetric strain increment is zero under undrained deformation (for details see e.g. Yu [35])

$\sigma_{r}=\sigma_{H}+\left(\sigma_{p}-\sigma_{H}\right)\left(\frac{r_{p}}{r}\right)^{2}$

$\sigma_{\theta}=\sigma_{H}+\left(\sigma_{p}-\sigma_{H}\right)\left(\frac{r_{p}}{r}\right)^{2}$

$\sigma_{z}=\sigma_{V}$

$u_{r}=\frac{\sigma_{p}-\sigma_{H}}{2 G_{0}} \frac{r_{p}^{2}}{r}$

where $\sigma_{p}$ is the total radial stress at the elasto/plastic boundary, $\sigma_{H}$ and $\sigma_{V}$ are the total horizontal and vertical stresses, respectively. 


\section{List of symbols}

a Radius of the cylindrical cavity

$c_{u} \quad$ Undrained shear strength

$c_{u, T X} \quad$ Undrained shear strength for triaxial compression conditions

$c_{u, P S} \quad$ Undrained shear strength for plane strain conditions

d Incremental operator

D Elastic stiffness matrix

e Void ratio

$e_{M} \quad$ Void ratio at critical state

$f_{y} \quad$ Function of the yield surface

$G \quad$ Shear modulus

$K_{0 \mathrm{NC}}$ Coefficient of lateral earth pressure at rest in normally consolidated conditions

$K_{0} \quad$ Coefficient of lateral earth pressure

M Slope of the critical state line

$p^{\prime} \quad$ Mean effective stress

$p^{\prime}{ }_{m} \quad$ Preconsolidation pressure

$q \quad$ Deviatoric stress

$\bar{q} \quad$ Invariant for anisotropic models. Radius of the yield surface in $\pi$-plane

$Q \quad$ Invariant for anisotropic models: $Q=\frac{2}{3} \bar{q}^{2}$

$R \quad$ Isotropic overconsolidation ratio

$s \quad$ Deviatoric stress

$u_{r} \quad$ Radial displacement

$v \quad$ Specific volume 
a Fabric tensor

$\alpha \quad$ Inclination of the yield surface

$\boldsymbol{\alpha}_{\mathbf{d}}$ Deviatoric fabric tensor

$\Lambda \quad$ Plastic multiplier

$\varepsilon \quad$ Strain scalar

$\varepsilon \quad$ Strain tensor

$d \varepsilon_{v} \quad$ Change in volumetric strain $d \varepsilon_{v}=d \varepsilon_{r}+d \varepsilon_{\theta}+d \varepsilon_{\theta}$

$d \varepsilon_{d} \quad$ Change in deviatoric strain $d \varepsilon_{d}=\sqrt{\frac{2}{3}\left\{d \boldsymbol{\varepsilon}_{d}\right\}^{T} \cdot\left\{d \boldsymbol{\varepsilon}_{d}\right\}}$

$\eta \quad$ Stress ratio: $\eta=q / p^{\prime}$ or $\boldsymbol{\eta}=\boldsymbol{\sigma}_{\mathbf{d}} / p^{\prime}$ (tensor)

$\theta \quad$ Lode's angle: $\theta=\tan ^{-1}\left[\frac{1}{\sqrt{3}}\left(2 \frac{\sigma_{2}^{\prime}-\sigma_{3}^{\prime}}{\sigma_{1}^{\prime}-\sigma_{3}^{\prime}}-1\right)\right]$

$\kappa \quad$ Slope of swelling line from $v-\ln p^{\prime}$ space

$\lambda \quad$ Slope of post yield compression line from $v-\ln p^{\prime}$ space

$v \quad$ Poisson's ratio

$\sigma, \sigma^{\prime}$ Total and effective stresses

$\sigma_{a} \quad$ Internal cavity pressure

$\sigma_{p} \quad$ Total radial stress at the elastic/plastic boundary

$\boldsymbol{\sigma}_{\boldsymbol{d}}^{\prime}$ deviatoric stress tensor

$\phi \quad$ Friction angle

$\omega, \omega_{d}$ Absolute and relative effectiveness of rotational hardening

CS Critical state

CSL Critical state line 
ESP Effective stress path

FEM Finite element method

MCC Modified cam clay

OCR Overconsolidation ratio

RH Rotational hardening

YS Yield surface

Subscripts/superscripts:

Initial

$d, v \quad$ deviatoric, volumetric

$H, V \quad$ horizontal, vertical

$i \quad$ any of the axis components $r, \theta, z$

$p \quad$ plastic

$r, \theta, z \quad$ cylindrical coordinates

Bold notation is used for tensors.

Compressive stresses and strains are assumed as positive because it is the conventional sign notation for geomaterials. 


\section{Table captions}

Table 1. Soil properties, Boston Blue clay.

Table 2. Soil state parameters, Boston Blue clay. 


\section{Figure captions}

Figure 1. Geometry of cylindrical cavity expansion: (a) cylindrical cavity; (b) horizontal cross section.

Figure 2. Yield curve of the S-CLAY1 model using triaxial invariants and visualization of new invariant $\bar{q}$.

Figure 3. Justification of new invariant $\bar{q}$ in $\pi$-plane.

Figure 4. Solution procedure for solving ordinary differential equations of cylindrical cavity expansion in GNU Octave.

Figure 5. Finite element model for cylindrical cavity expansion.

Figure 6. Stress distributions around the cavity: (a) $\mathrm{OCR}=1$; (b) $\mathrm{OCR}=1.5$; (c) $\mathrm{OCR}=5$.

Figure 7. Variation of radial stress and excess pore pressure at cavity wall during cavity expansion (ambient pore pressures not included).

Figure 8. $p^{\prime}-q$ stress paths at cavity wall: (a) $\mathrm{OCR}=1$; (b) $\mathrm{OCR}=1.5$; (c) $\mathrm{OCR}=5$.

Figure 9. Stress paths at cavity wall in $\pi$-plane: (a) $\mathrm{OCR}=1$; (b) $\mathrm{OCR}=1.5$; (c) $\mathrm{OCR}=5$.

Figure 10. Changes in fabric anisotropy: (a) $\mathrm{OCR}=1$; (b) $\mathrm{OCR}=1.5$; (c) $\mathrm{OCR}=5$.

Figure 11. Visualization of the changes in fabric anisotropy $(\mathrm{OCR}=1)$.

Figure 12. Influence of initial fabric anisotropy $(\mathrm{OCR}=1)\left(c_{u}=69.6 \mathrm{kPa}\right.$ for $\alpha=0$ and $c_{u}=56.6 \mathrm{kPa}$ for $\left.\alpha=0.46\right)$.

Figure 13. Results for isotropic and anisotropic Cam clay models $(\mathrm{OCR}=1)\left(c_{u}=55.5\right.$ $\mathrm{kPa}$ for MCC and $c_{u}=56.6 \mathrm{kPa}$ for S-CLAY1S).

Figure 14. Comparison of stress paths at cavity wall with and without rotational hardening ( $\mathrm{OCR}=1)$ : (a) in $\pi$-plane; (b) p'- $q$ diagram.

Figure 15. Stress distributions around the cavity with and without rotational hardening $(\mathrm{OCR}=1)$. 


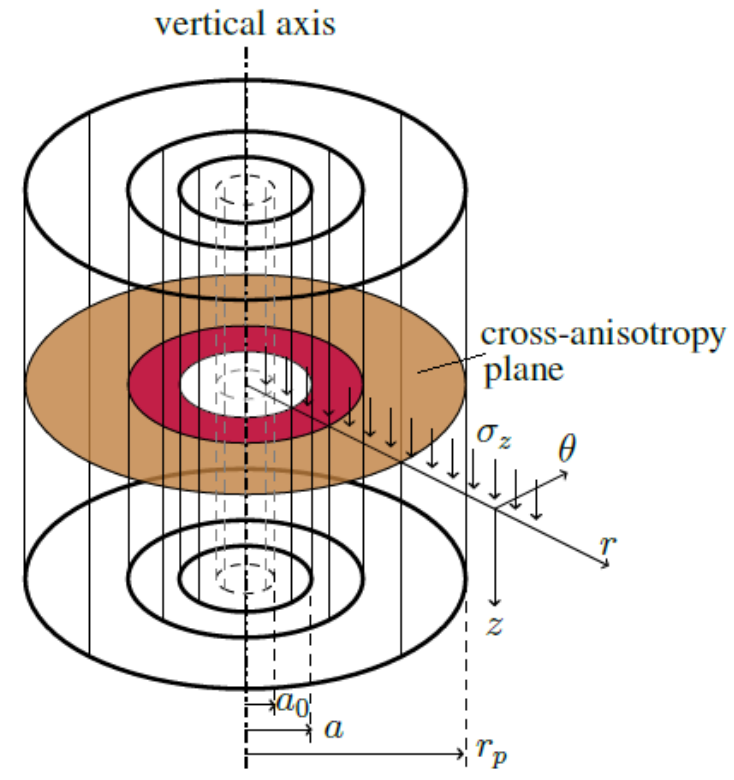

(a)

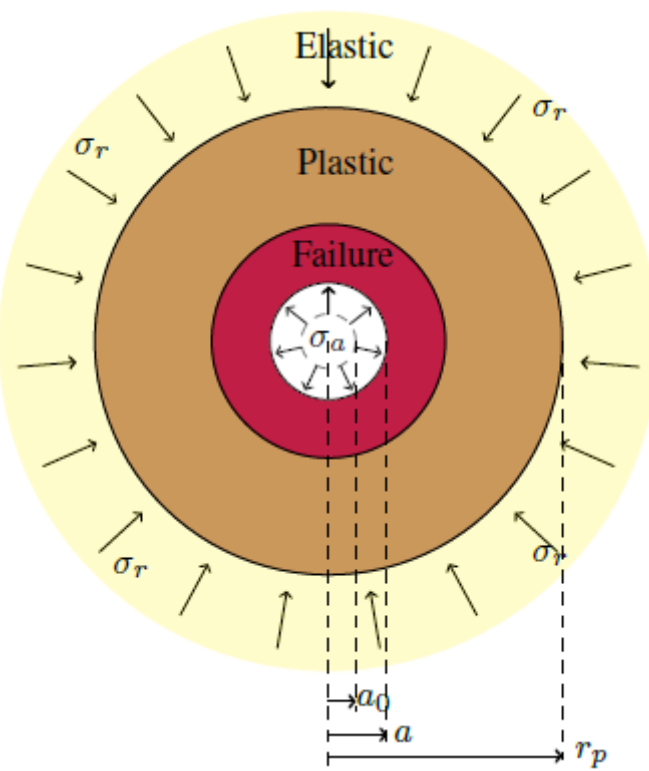

(b)

Figure 1. Geometry of cylindrical cavity expansion: (a) cylindrical cavity; (b) horizontal (cross-anisotropy) section.

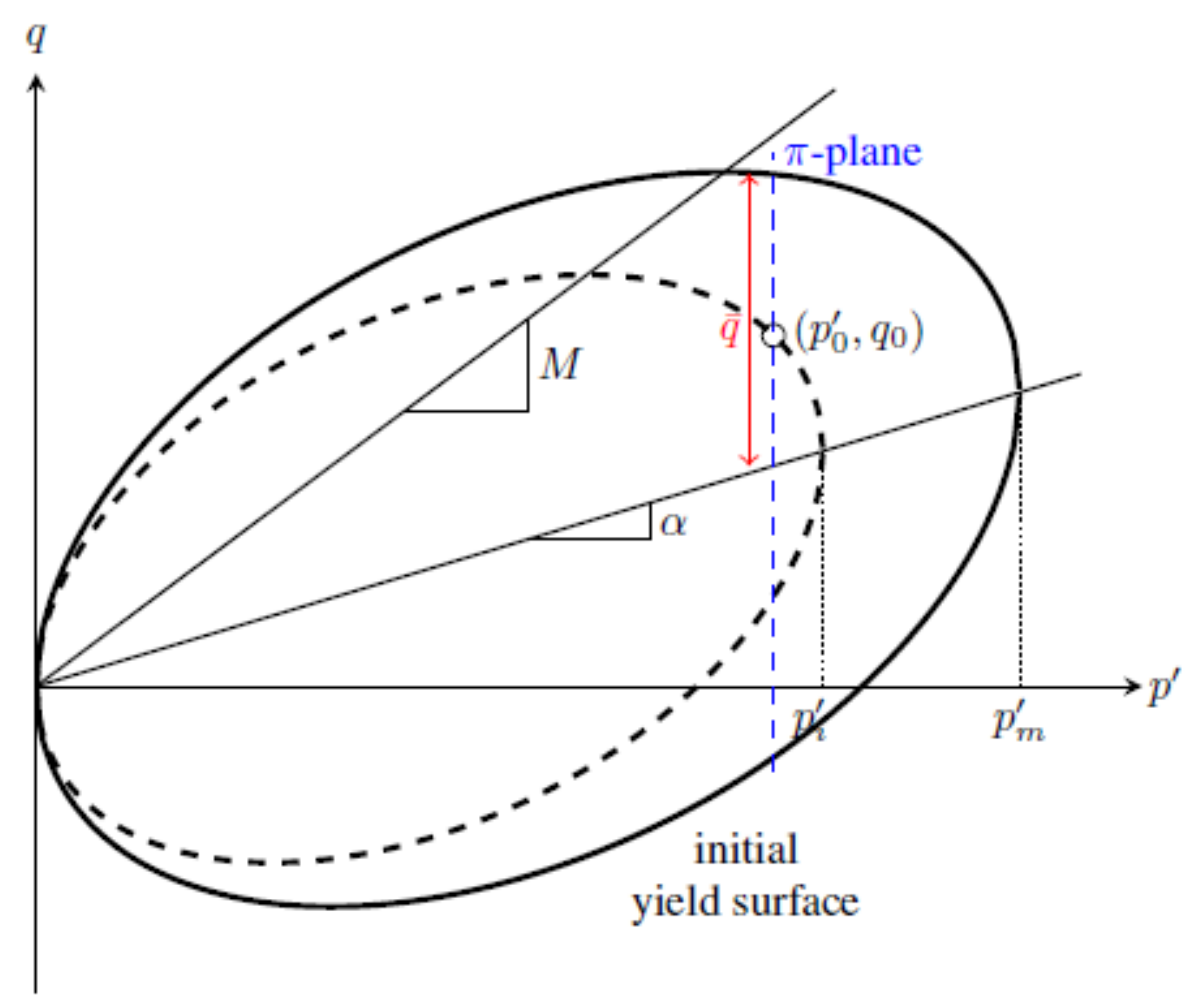

Figure 2. Yield curve of the S-CLAY1 model using triaxial invariants and visualization of new invariant $\bar{q}$. 


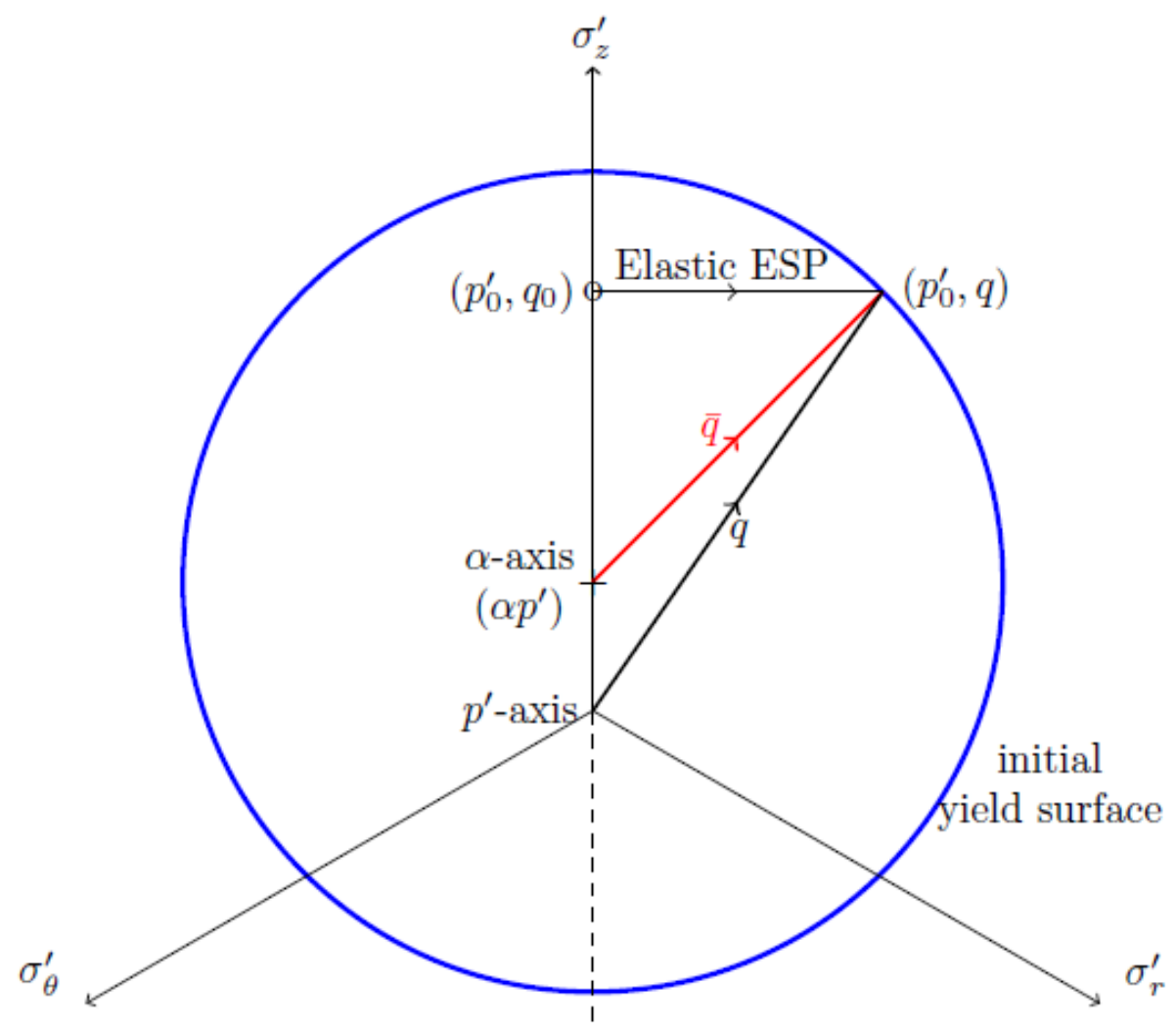

ESP: Effective stress path

Figure 3. Justification of new invariant $\bar{q}$ in $\pi$-plane. 
1. Data: initial stress state, model parameters and $a / a_{0}$

2. Set initial anisotropic components $\left(\alpha_{r}, \alpha_{\theta}\right.$ and $\left.\alpha_{z}\right)$

3. Determine stresses at initial yield $\left(\sigma_{r p}^{\prime}, \sigma_{\theta p}^{\prime}\right.$, and $\left.\sigma_{z p}^{\prime}\right)$

4. Determine elasto/plastic boundary $\left(r_{p}\right)$

$5 \quad$ Set initial $r_{x} / a=1$

6. $\quad$ While $(i \leq n s t e p)$

Solve $r_{x p} / a$ for given $a / a_{0}, r_{x} / a, \sigma_{r p}^{\prime}, \sigma_{\theta p}^{\prime}, \sigma_{z p}^{\prime}$ and $G_{0}$

Set interval $\left\{R_{x} / a\right\}=$ linspace $\left(r_{x p} / a, r_{x} / a\right.$, inc. $)$

Solve partial differential equation:

$Y(\sigma, \alpha)=1 \operatorname{sode}\left(" O D E^{\prime \prime},\left(\sigma_{r p}^{\prime}, \sigma_{\theta p}^{\prime}, \sigma_{z p}^{\prime}, \alpha_{r}, \alpha_{\theta}, \alpha_{z}\right),\left\{R_{x} / a\right\}\right)$

lsode:

7.

function $y d o t=O D E\left(Y,\left\{R_{x} / a\right\}\right)$

7.1. Obtain:

$\left[\begin{array}{llllll}\sigma_{r}^{\prime} & \sigma_{\theta}^{\prime} & \sigma_{z}^{\prime} & \alpha_{r} & \alpha_{\theta} & \alpha_{z}\end{array}\right]=Y$ model parameters

7.2. Calculate:

$\alpha_{i}^{d}$ for $i=r, \theta, z$ and $\alpha$

$p^{\prime}, G, E^{\prime}$

$\frac{\partial f_{y}}{\partial \sigma_{i}^{\prime}}$ for $i=r, \theta, z$ and hardening modulus $\mathcal{H}$

$c_{11}, c_{12}, c_{13}, c_{22}, c_{23}, c_{33}$ and $\Gamma$

$\frac{d \alpha_{i}^{d}}{d r}$ for $i=r, \theta, z$

7.3 Compute

$d \sigma_{r}^{\prime} /_{d r}, d \sigma_{\theta}^{\prime} /_{d r}$ and $d \sigma_{z}^{\prime} /_{d r}$

$d \alpha_{r}^{d} /_{d r}, d \alpha_{\theta}^{d} /_{d r}$ and $d \alpha_{z}^{d} /_{d r}$

\section{endfunction}

8.

Update $r_{x} / a \leftarrow r_{x} / a+\Delta r / a\left(\Delta r / a=\left(r_{p} / a-1\right) /\right.$ nstep $)$

$$
i \leftarrow i+1
$$

9.

Get $\sigma_{r}^{\prime}, \sigma_{\theta}^{\prime}, \sigma_{z}^{\prime}, \alpha_{r}, \alpha_{\theta}$ and $\alpha_{z}$ from $Y(\sigma, \alpha)$

\section{End}

10. Calculate excess pore pressure $\Delta u$

11. If $(\mathrm{OCR}>1)$ Calculate elastic stresses

End

Figure 4. Solution procedure for solving ordinary differential equations of cylindrical cavity expansion in GNU Octave. 


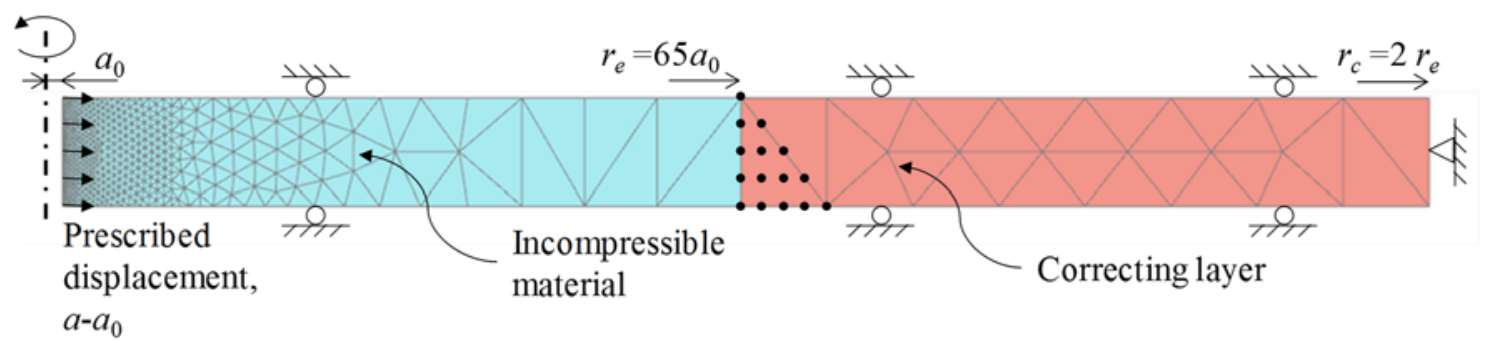

Figure 5. Finite element model for cylindrical cavity expansion. 


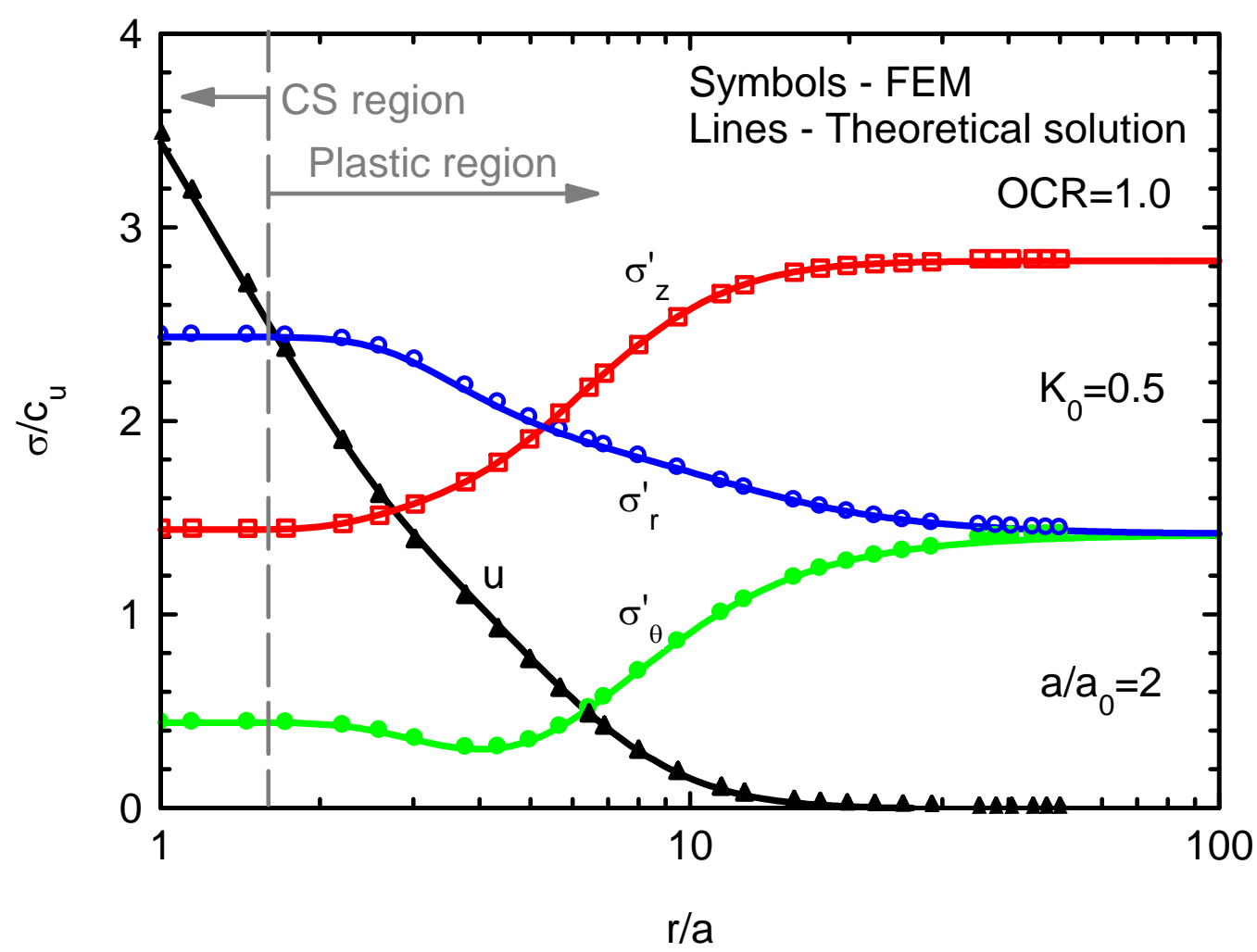

(a)

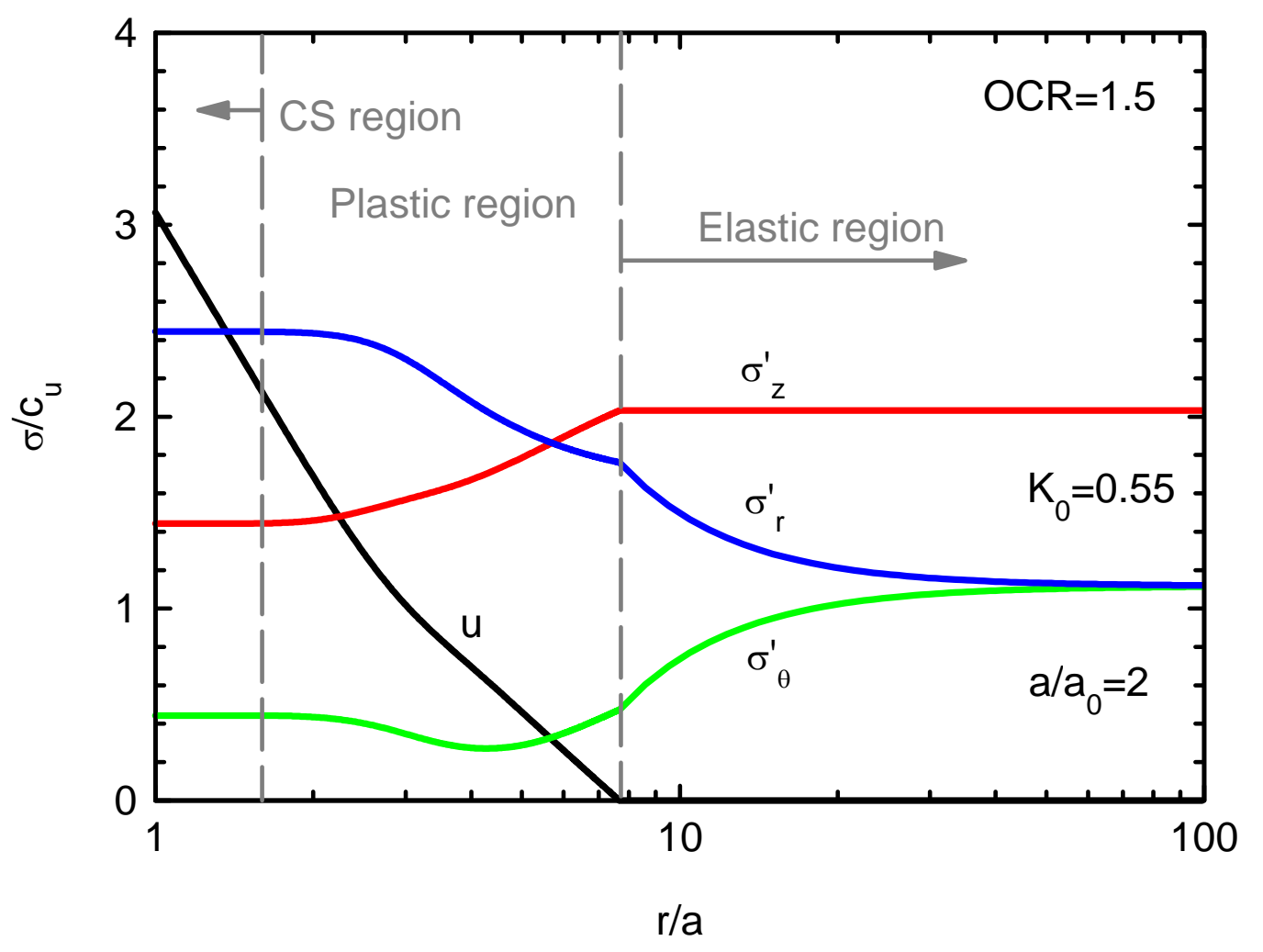

(b) 


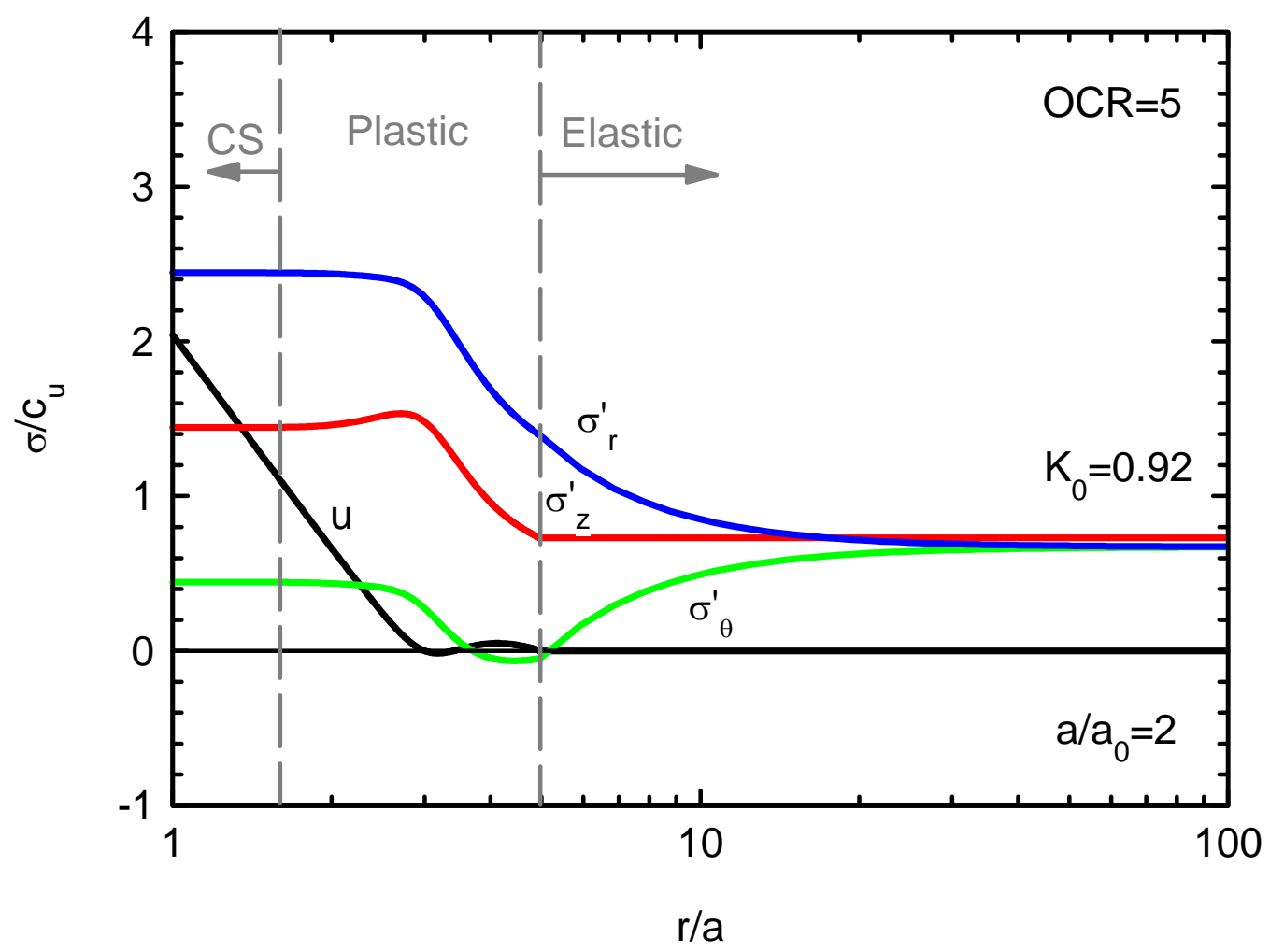

(c)

Figure 6. Stress distributions around the cavity: (a) $\mathrm{OCR}=1$; (b) $\mathrm{OCR}=1.5$; (c) $\mathrm{OCR}=5$. 


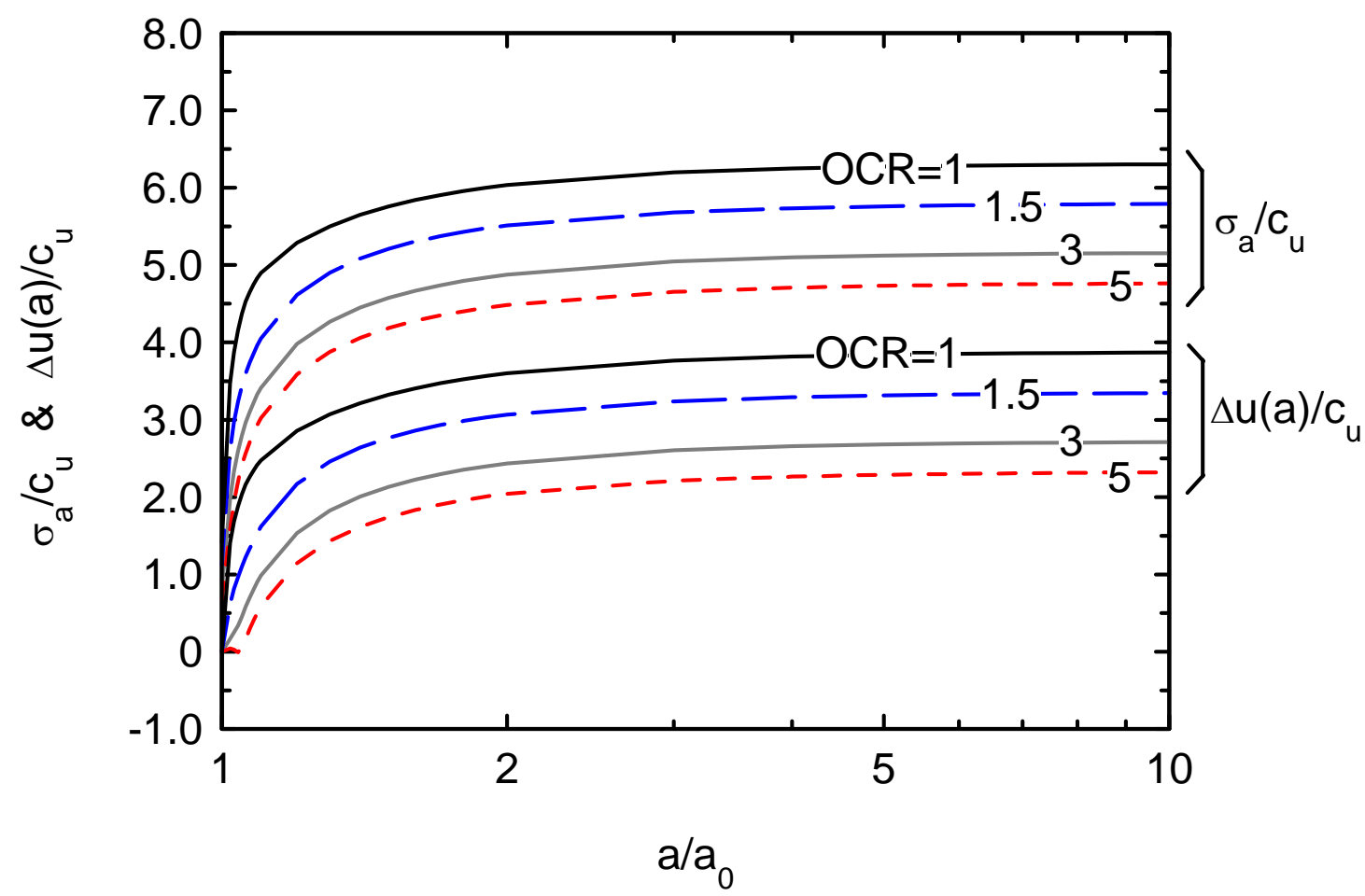

Figure 7. Variation of radial stress and excess pore pressure at cavity wall during cavity expansion (ambient pore pressures not included). 


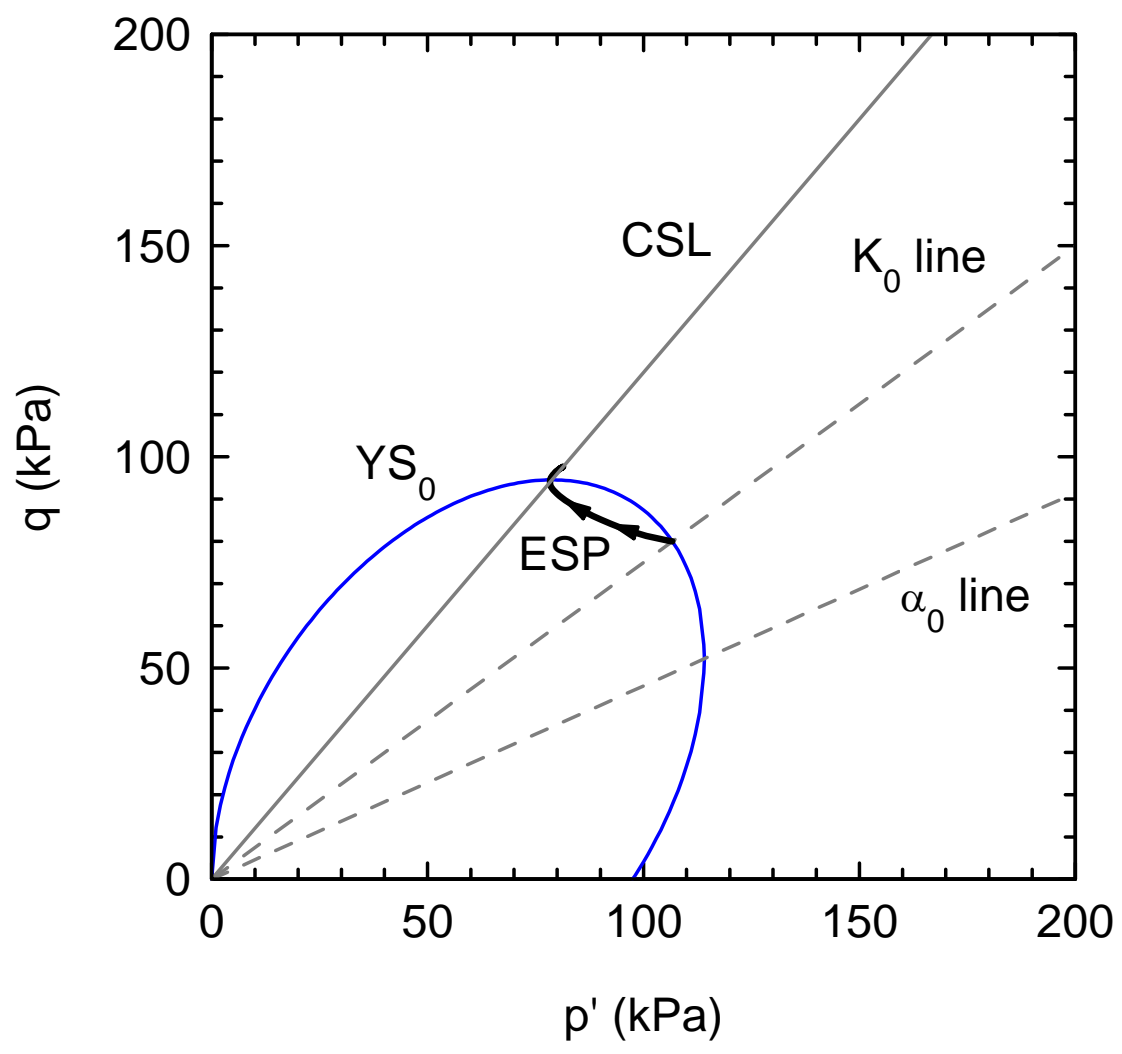

(a)

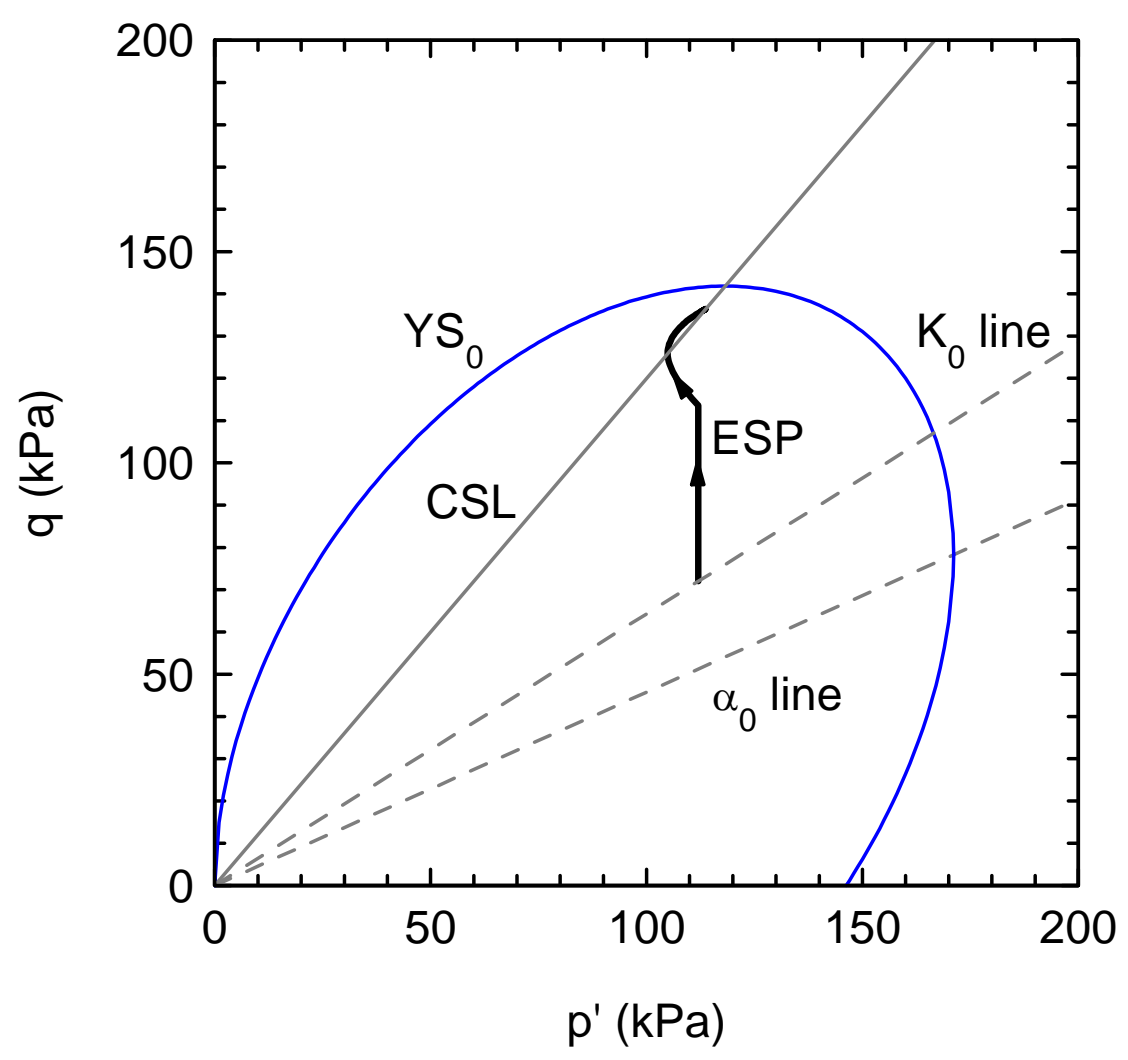

(b) 


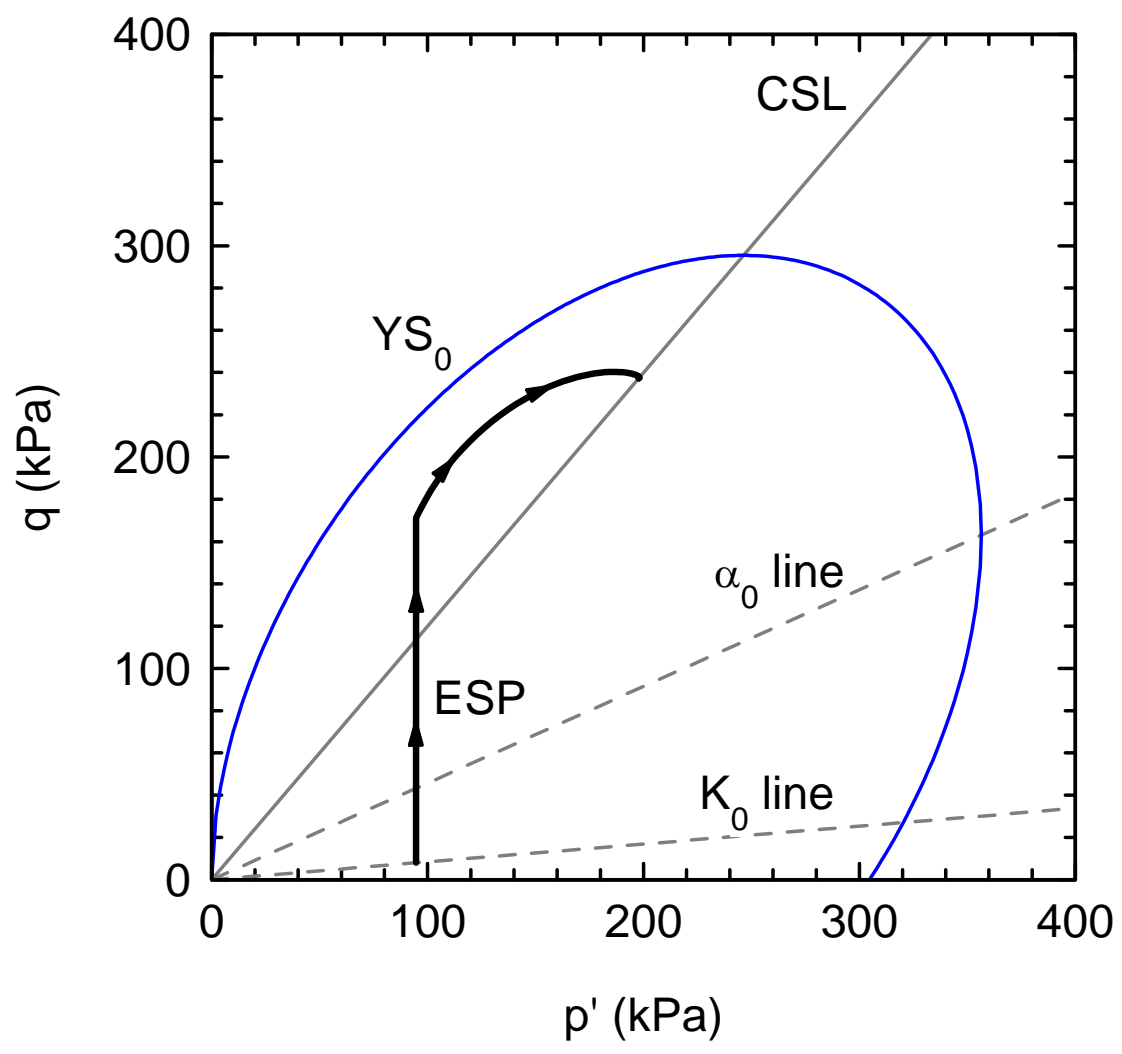

(c)

ESP: Effective stress path

CSL: Critical state line

$\mathrm{YS}_{0}$ : Initial Yield Surface in triaxial plane

Figure 8. $p^{\prime}-q$ stress paths at cavity wall: (a) $\mathrm{OCR}=1$; (b) $\mathrm{OCR}=1.5$; (c) $\mathrm{OCR}=5$. 


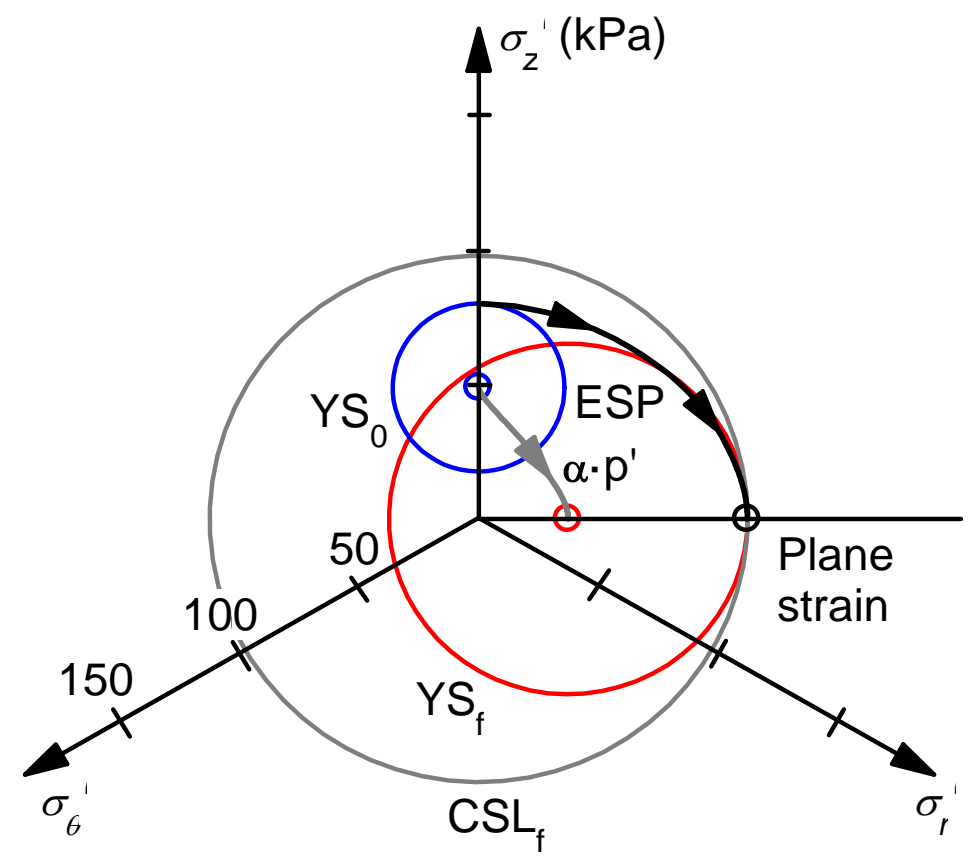

(a)

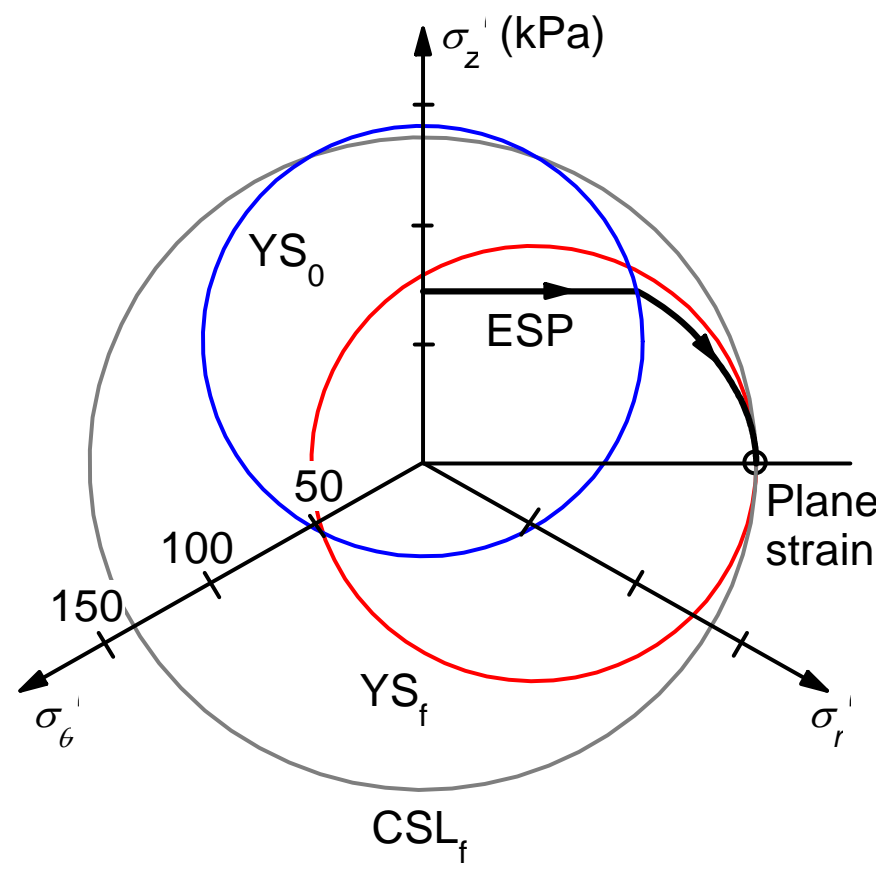

(b) 


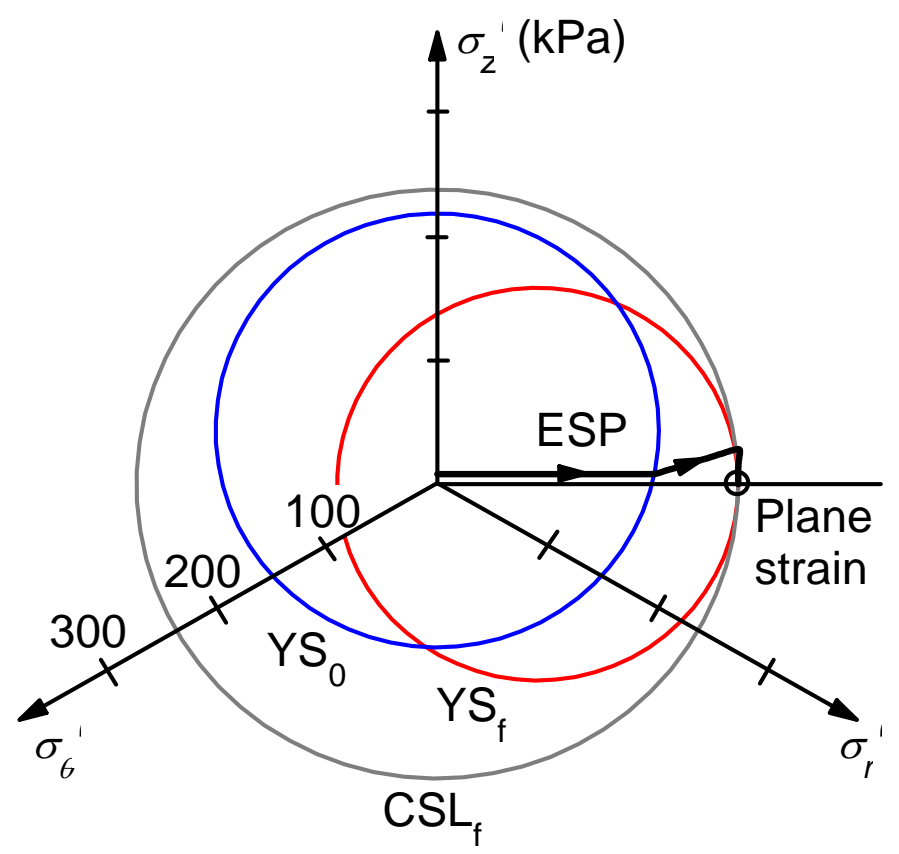

(c)

ESP: Effective stress path

CSLf: Critical State Surface at failure

$\mathrm{YS}_{0}$ : Initial Yield Surface

YSf: Final Yield Surface

Figure 9. Stress paths at cavity wall in $\pi$-plane: (a) $\mathrm{OCR}=1$; (b) $\mathrm{OCR}=1.5$; (c) $\mathrm{OCR}=5$. 


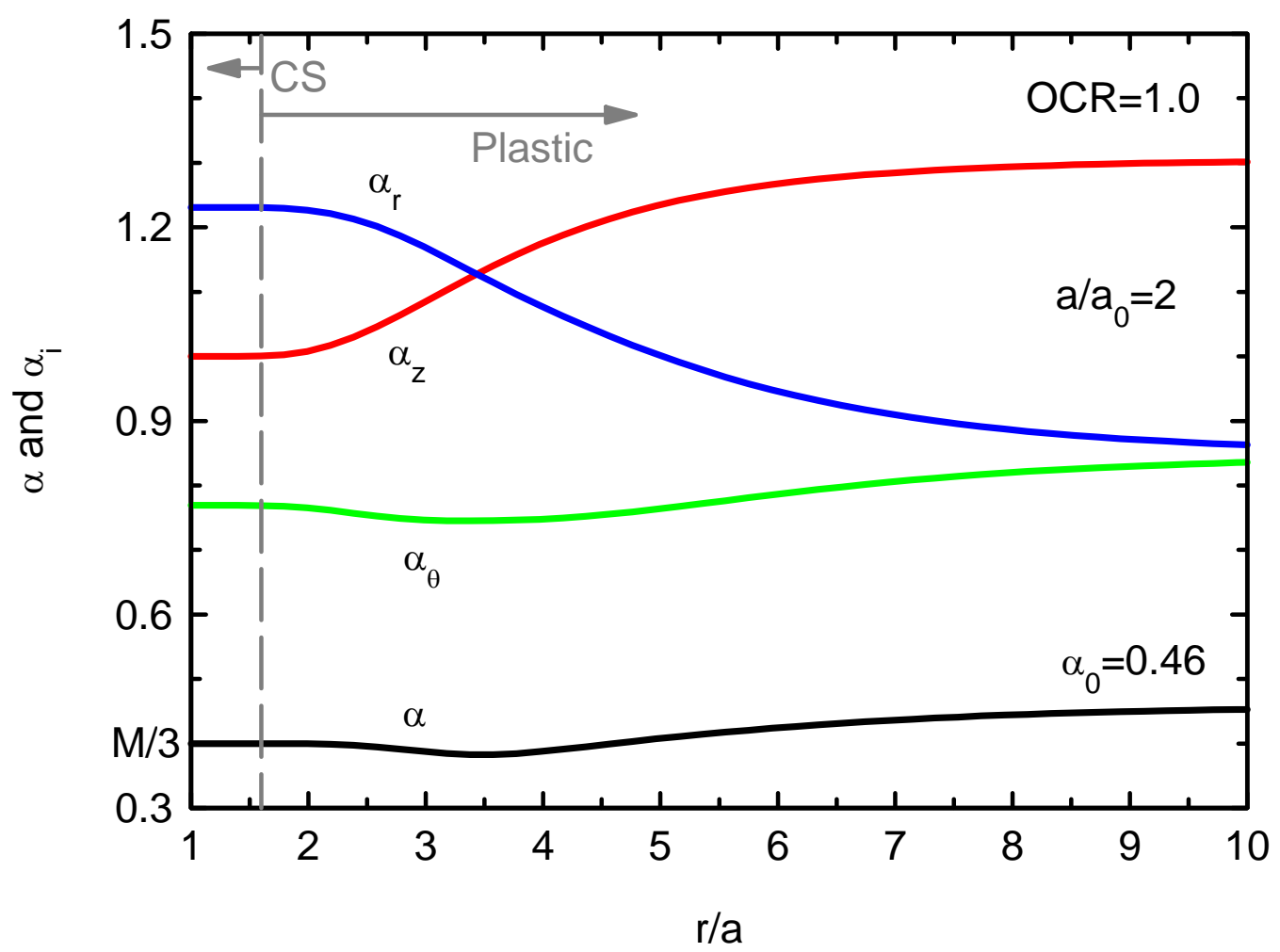

(a)

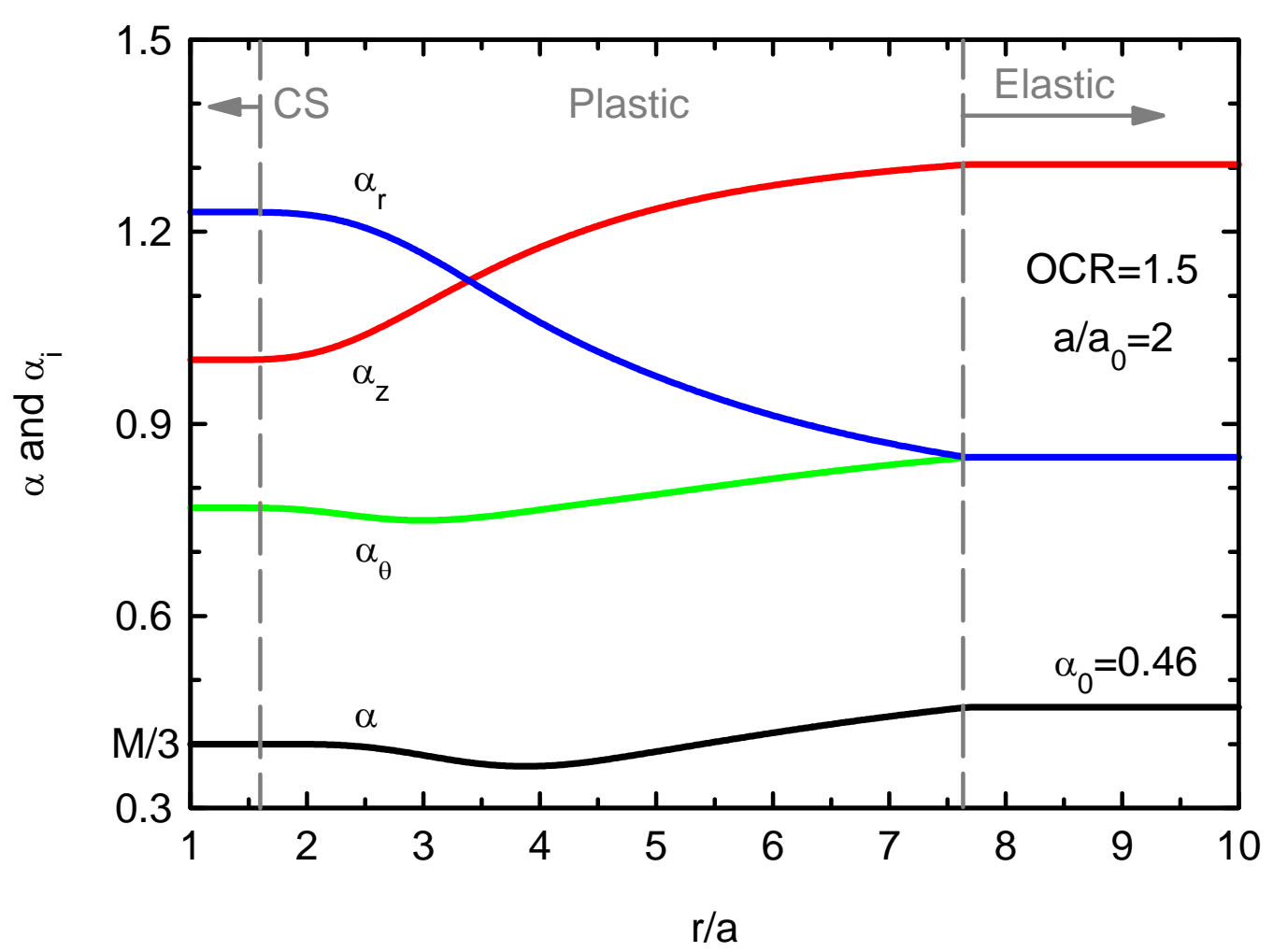

(b) 


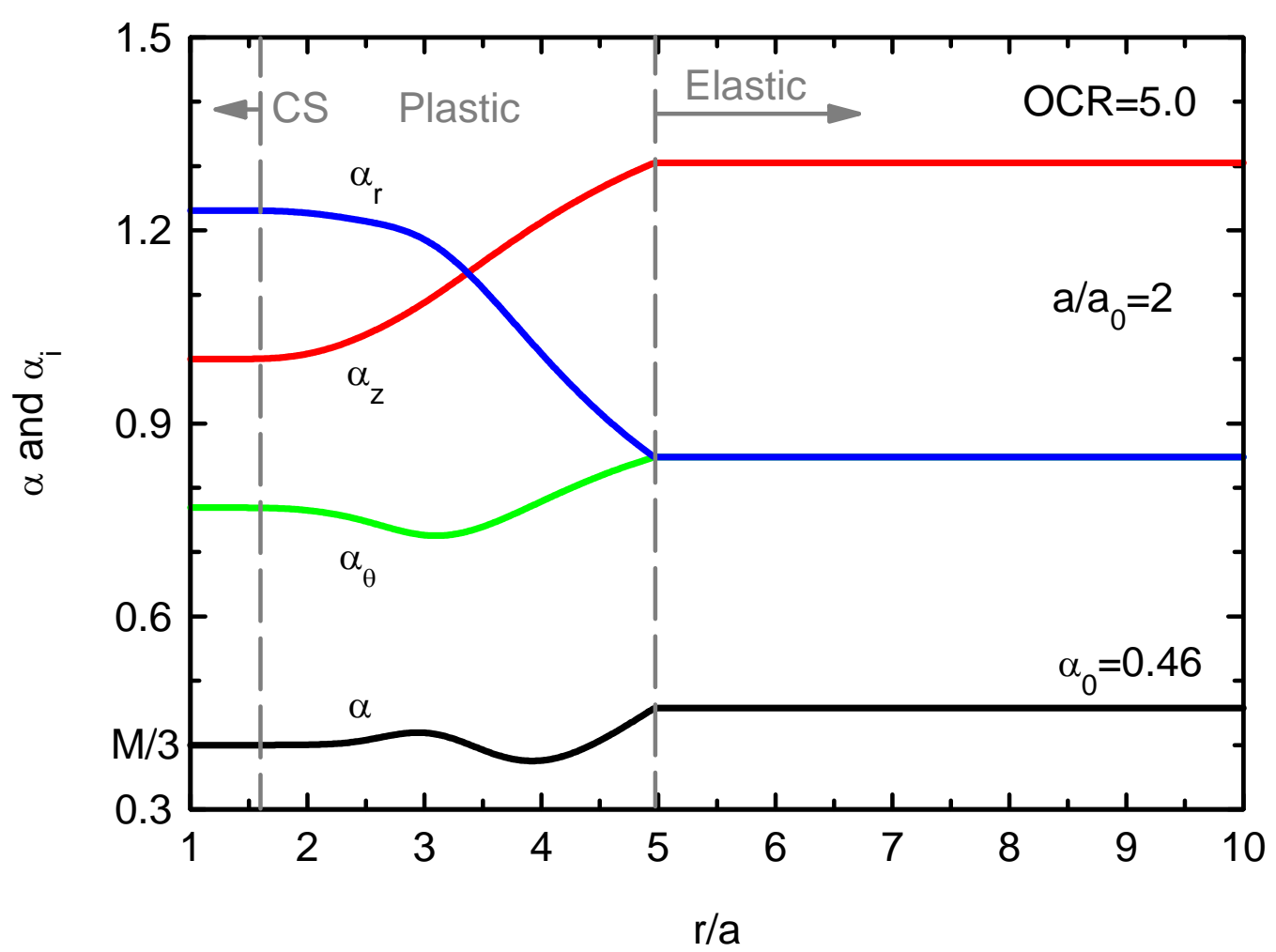

(c)

Figure 10. Changes in fabric anisotropy: (a) $\mathrm{OCR}=1$; (b) $\mathrm{OCR}=1.5$; (c) $\mathrm{OCR}=5$. 

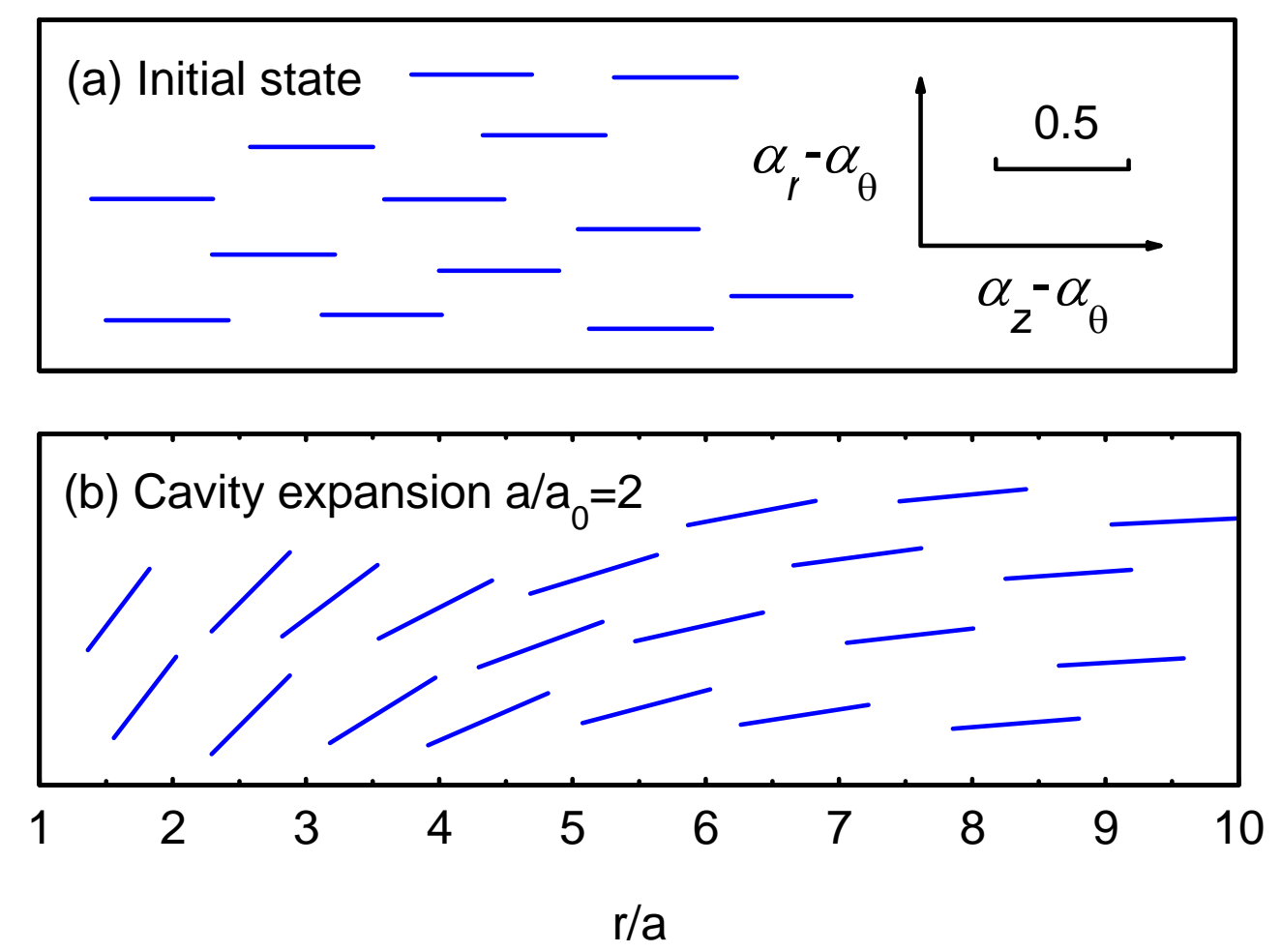

Figure 11. Visualization of the changes in fabric anisotropy $(\mathrm{OCR}=1)$. 


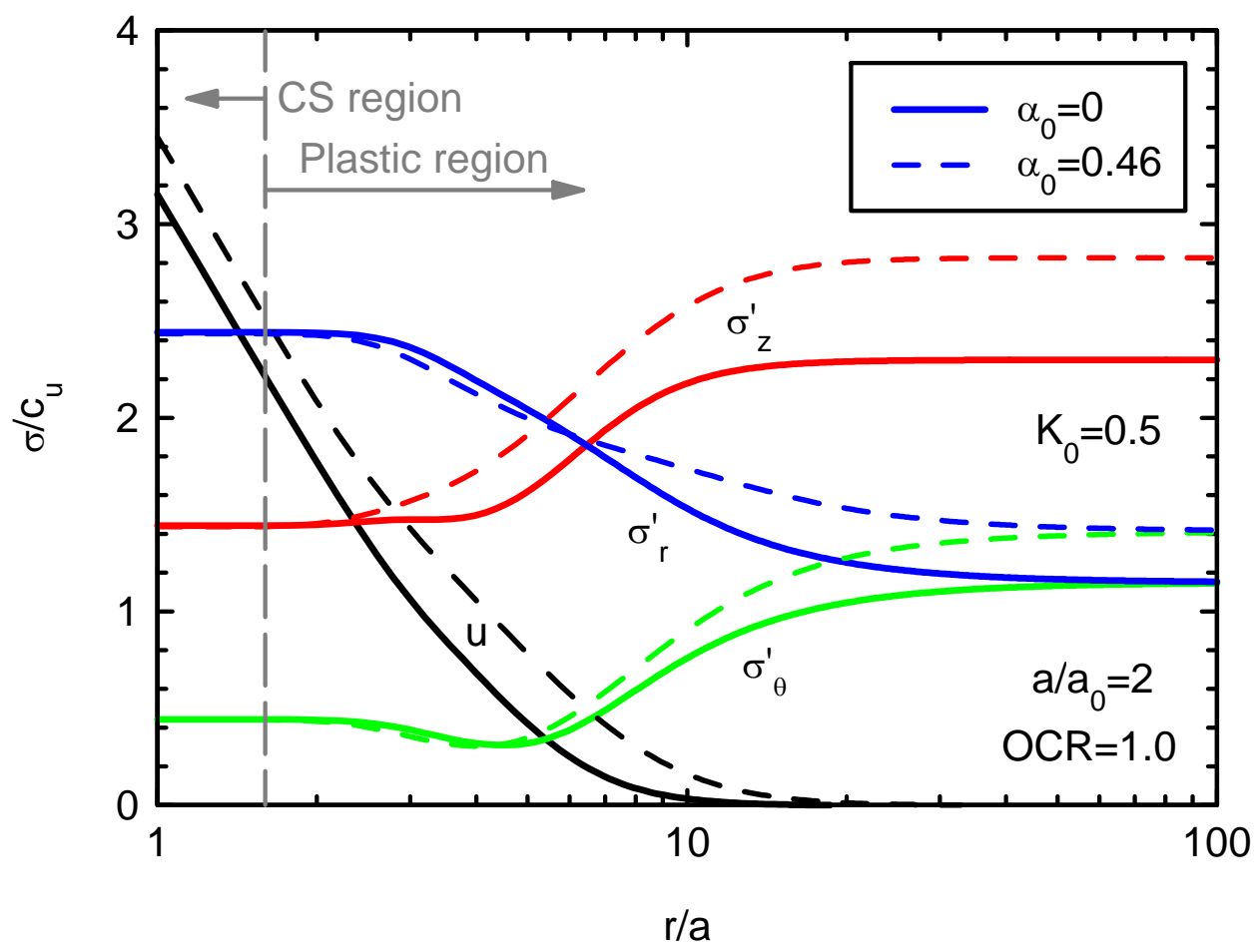

Figure 12. Influence of initial fabric anisotropy $(\mathrm{OCR}=1)\left(c_{u}=69.6 \mathrm{kPa}\right.$ for $\alpha=0$ and $c_{u}=56.6 \mathrm{kPa}$ for $\left.\alpha=0.46\right)$.

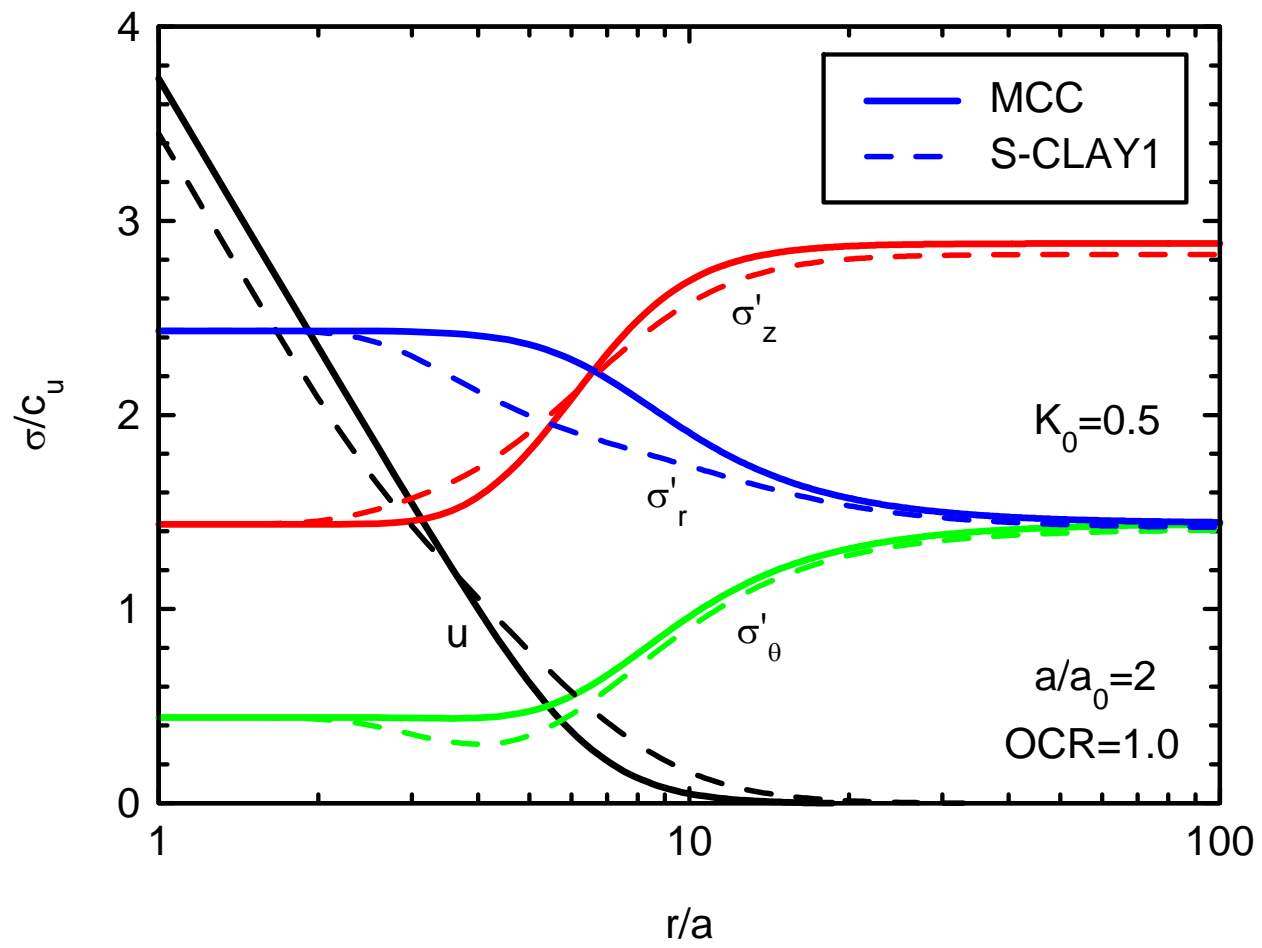

Figure 13. Results for isotropic and anisotropic Cam clay models $(\mathrm{OCR}=1)\left(c_{u}=55.5\right.$ $\mathrm{kPa}$ for MCC and $c_{u}=56.6 \mathrm{kPa}$ for S-CLAY1S). 


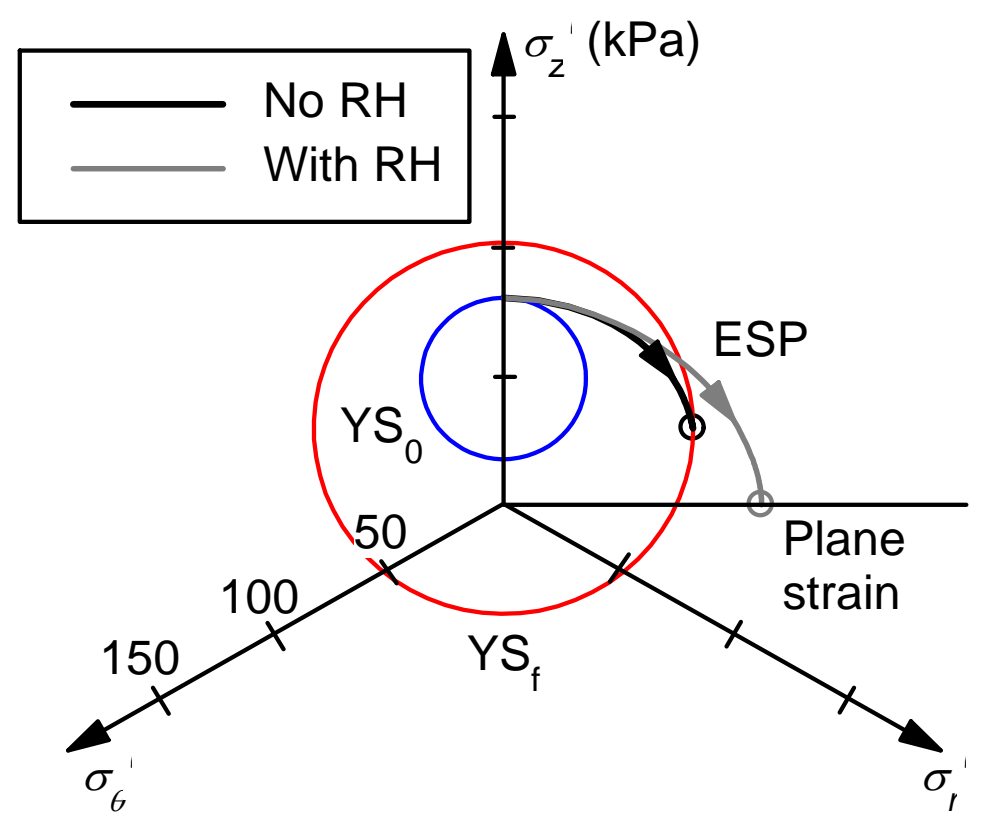

(a)

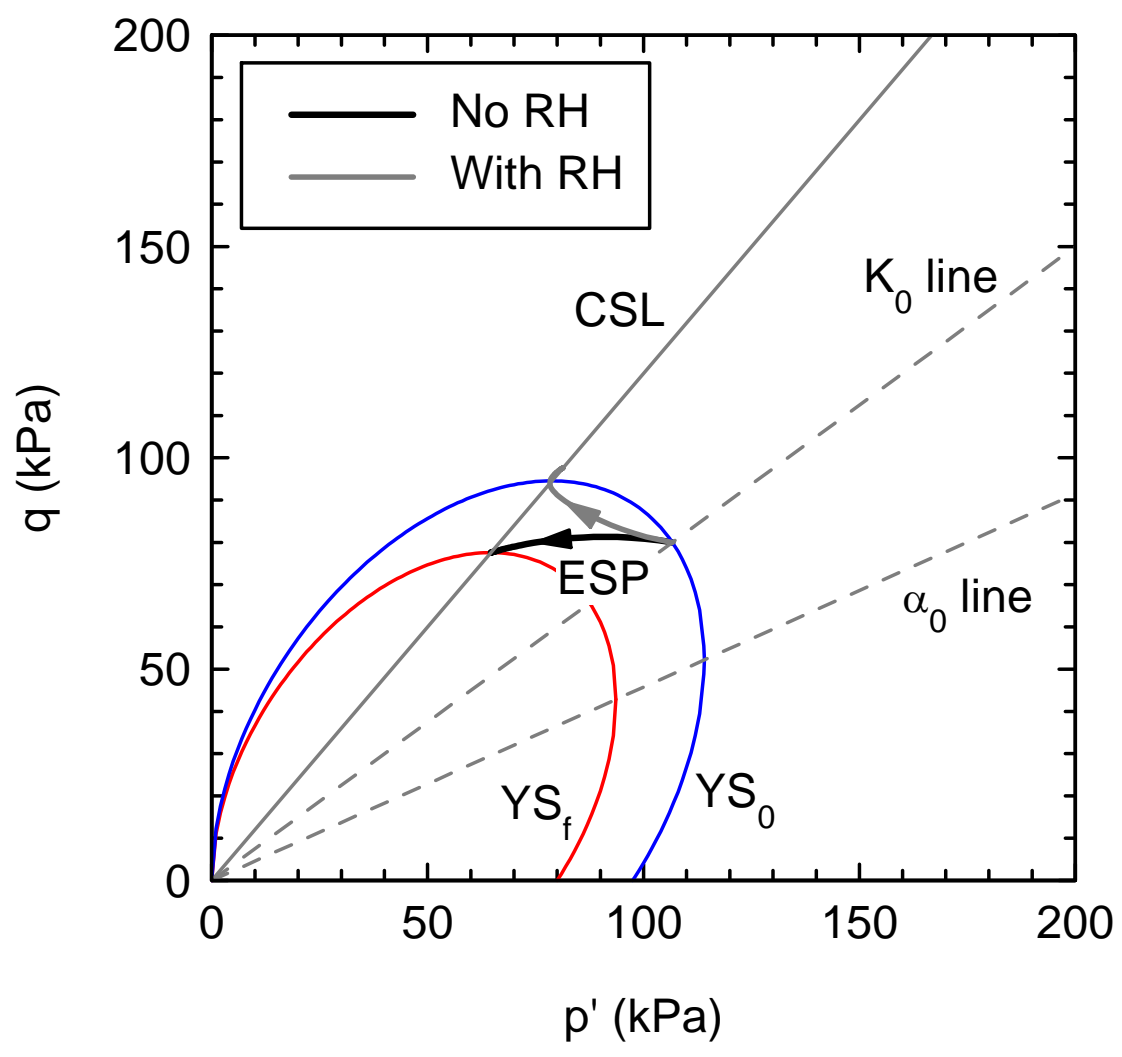

(b)

Figure 14. Comparison of stress paths at cavity wall with and without rotational hardening (OCR=1): (a) in $\pi$-plane; (b) $p^{\prime}-q$ diagram. 


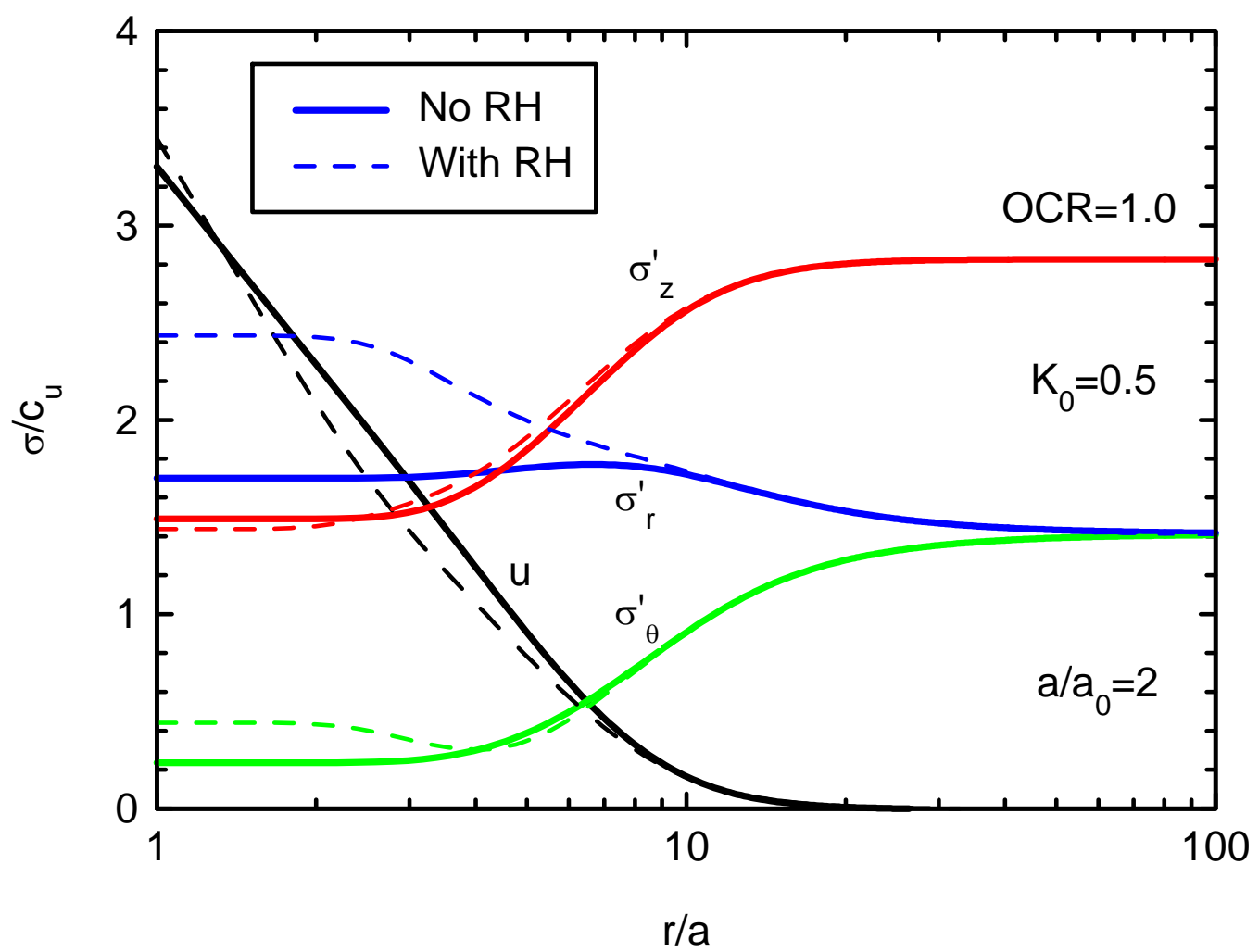

Figure 15. Stress distributions around the cavity with and without rotational hardening $(\mathrm{OCR}=1)$. 